\title{
A selective $p 38 \alpha / \beta$ MAPKs inhibitor alleviates neuropathology and cognitive impairment by modulating microglia function in 5XFAD mouse
}

\section{Min Sung Gee}

Kyung Hee University

\section{Seung Hwan Son}

Kyung Hee University

\section{Seung Ho Jeon}

Kyung Hee University

Jimin Do

Kyung Hee University

Namkwon Kim

Kyung Hee University

\section{Yeon-Joo Ju}

Kyung Hee University

\section{Soo Jin Lee}

Kyung Hee University

\section{Eun Kyoung Chung}

Kyung Hee University

Kyung-Soo Inn

Kyung Hee University

Nam-Jung Kim

Kyung Hee University

Jong Kil Lee ( $\square$ jklee3984@khu.ac.kr)

Kyung Hee University https://orcid.org/0000-0002-2428-3530

\section{Research}

Keywords: Alzheimer's disease, Amyloid- $\beta$, P38 mitogen-activated protein kinase, Kinase inhibitor, Microglia

Posted Date: February 25th, 2020

DOI: https://doi.org/10.21203/rs.2.21305/v2 
License: (c) (i) This work is licensed under a Creative Commons Attribution 4.0 International License. Read Full License

Version of Record: A version of this preprint was published at Alzheimer's Research and Therapy on April 21 st, 2020. See the published version at https://doi.org/10.1186/s13195-020-00617-2. 


\section{Abstract}

Background: Chronic neuroinflammation, aggressive amyloid beta $(A \beta)$ deposition, neuronal cell loss and cognitive impairment are pathological symptoms of Alzheimer's disease (AD). Regarding these symptoms, resolution of neuroinflammation and inhibition of $A \beta$-driven pathology might be one of the important strategies for $A D$ therapy. Previous efforts to prevent $A D$ progression have identified that $p 38$ mitogen-activated protein kinase (MAPK) is a promising target for AD therapy. Especially, recent studies showed that pharmacological p38a MAPK inhibition improved memory impairment in AD mouse models.

Methods: In this study, we explored the therapeutic potential of NJK14047, a selective p38a/ $\beta$ MAPKs inhibitor, using an Alzheimer's disease mouse model, 5XFAD. The mice were injected $2.5 \mathrm{mg} / \mathrm{kg}$ NJK14047 or vehicle every other day for 3 months. Morris water maze task and histological imaging analysis were performed. Protein and mRNA expression levels were measured using immunoblotting and qRT-PCR respectively. In in vitro studies, the cytotoxicity of microglial conditioned medium and astrocyte conditioned medium on primary neurons were measured using MTT assay and TUNEL assay.

Results: NJK14047 treatment downregulated phospho-p38 MAPK levels, decreased the amount of A $\beta$ deposits, and prevented spatial learning memory loss in 9-month-old 5XFAD mice. Interestingly, we found the decreased pro-inflammatory conditions and increased expression of alternatively activated microglial markers and microglial phagocytic receptors. Furthermore, NJK14047 treatment reduced the number of Fluoro-jade B positive cells, a class of degenerating neurons, in the brains of 5XFAD mice. The neuroprotective effect of NJK14047 was further confirmed by in vitro studies.

Conclusion: Taken together, a selective $p 38 \alpha / \beta$ MAPKs inhibitor NJK14047 successfully showed therapeutic effects in 5XFAD mice. Our data support that p38 MAPKs inhibition is a potential strategy for AD therapy and NJK14047 might be one of the promising candidates for AD therapeutics targeting p38 MAPKs.

\section{Background}

Alzheimer's disease (AD) is a common progressive neurodegenerative disorder characterized by severe neuronal loss leading to cognitive dysfunction. The major neuropathological feature of $A D$ is the aggregation and deposition of amyloid $\beta(A \beta)$ peptides. At present, no effective drug or treatment has been developed for the prevention or remedy of this disease [1-3]. Aggregated $A \beta$ peptides and their deposition are considered one of the major risk factors and causes of $A D[3,4]$. Aggregated $A \beta$ peptides play critical roles in neuronal degeneration, neuroinflammation, and oxidative stress $[5,6]$. Thus, therapeutic approaches for the efficient removal of $A \beta$ deposits may provide neuroprotective benefits in $A D$.

Recently, a series of reports have demonstrated that neuroinflammation, in addition to $A \beta$ peptides, may exacerbate AD. Therefore, the modulation of neuroinflammation may represent an important therapeutic strategy for AD [6-8]. Neuroinflammation is mainly mediated by microglial cells, which are resident 
immune cells in the central nervous system (CNS) [6, 9]. Under physiological conditions, microglial cells execute diverse functions, including the regulation of brain development, the maintenance of homeostasis, and the clearance of old synapses or other debris, as well as $A \beta$ peptides. However, in AD, microglia has been known to be chronically activated, resulting in impaired $A \beta$ clearance and overexpression of pro-inflammatory signals, which are related to neurotoxicity $[10,11]$. The regulation of microglial activation therefore represents a potential therapeutic strategy. Microglial activation may be regulated by lowering excessive pro-inflammatory immune chemotaxis and enhancing neuroprotective functions.

Microglial activation involves several well-known pro-inflammatory signal cascades [12], including those mediated by mitogen-activated protein kinase (MAPK). The MAPK family is a family of serine/threonine protein kinases that regulates cell properties in response to extracellular stimuli such as growth factors and inflammatory cytokines [13]. P38 MAPKs, one of the three MAPKs established in mammalian cells, are mainly activated by inflammatory cytokines and environmental stresses [14]. P38 MAPKs have four isoforms $\alpha, \beta, y$ and $\delta$ which can be divided into two subgroups; one is $p 38 a$ and $p 38 \beta$ and the other is p38 $y$ and p38ס [15]. Especially, p38a and p38 $\beta$ MAPKs are highly expressed in adult mouse brain [16]. SB203580, a traditional p38a/ $\beta$ MAPKs inhibitor, had shown therapeutic effects in LPS-induced depression model and AD mouse model $[17,18]$. Recently, two selective p38a MAPK inhibitors, neflamapimod (VX-745) and MW150, also showed therapeutic effects in aged rats and AD mouse model respectively $[19,20]$. In addition, neflamapimod had been entered into phase 2 a clinical trials and showed improvements in memory and learning in early AD patients $[21,22]$. These results suggest that $p 38 a / \beta$ MAPKs are important targets in $A D$ therapeutics and their inhibitors can be promising drugs.

In our previous study, we reported that a novel and selective p38a/ 3 MAPKs inhibitor, NJK14047, could successfully ameliorate microglia-mediated neuroinflammation [23]. It reduced LPS mediated inflammatory responses of BV2 cells and mouse model. For the extension of that study, we intended to investigate the potential of NJK14047 as a therapeutic agent for AD. In this study, we used five familial Alzheimer's disease (5XFAD) transgenic mice which have been generally used for AD mice model as well as APP/PS1 mice. 5XFAD mice overexpress human amyloid precursor protein (hAPP) with three FAD mutations; Swedish (K670N, M671L), Florida (1716V) and London (V717I) and human presenilin 1 (PS1) with two FAD mutations; M146L and L286V, under control of neuron-specific Thy 1 promotor [24]. Here, we administered NJK14047 to 5XFAD mouse model and found that NJK14047 could ameliorate memory loss, $A \beta$ deposition, neuroinflammation, and neuronal degeneration via the selective inhibition of $p 38 a / \beta$ MAPKs.

\section{Methods}

Chemicals and reagents. A selective $\mathrm{p} 38 \mathrm{\alpha} / \mathrm{\beta}$ MAPKs inhibitor NJK14047 was synthesized by a previously reported procedure (>97\%, HPLC) [23, 25]. Dulbecco's modified Eagle's medium (DMEM), Fetal bovine serum (FBS) and penicillin/streptomycin were purchased from GE healthcare HyClone ${ }^{\mathrm{Tm}}$. Cell culture flasks and plates were purchased from SPL $(70075,30006,30024,30048,30096)$. Lipopolysaccharide 
(LPS) from Escherichia coli serotype 055:B5 (L6529, 2500,000 EU/mg), Methylthiazolyldiphenyltetrazolium bromide (MTT), Thioflavin S (T1892) and DPX mounting medium (06522) were obtained from Sigma-Aldrich. Neurobasal medium (21103-049), B27 supplements (17504-044), RIPA buffer (89901), protease and phosphatase inhibitor cocktail (78445) and Griess reagent (G7921) were obtained from ThermoFisher Scientific. Fluorescence mounting medium (S3023) was purchased from Dako. TUNEL (G3250) assay kits were obtained from Promega. Information about antibodies used in this study is listed in Table.1.

Animals and treatment. 5XFAD mice on B6/SJL background (34840-JAX, Tg6799) and wild type B6/SJL mice from Jackson Laboratory were bred and maintained in an individual ventilated cage, $12 \mathrm{~h} \mathrm{light/dark}$ cycle and $22{ }^{\circ} \mathrm{C}$ condition. 5XFAD mice were divided into two experimental groups (treatment group and vehicle group) using the block randomization method. Similar to previous studies [26, 27], treatment group was treated with NJK14047 at $2.5 \mathrm{mg} / \mathrm{kg}$ every other day from 6-month-old to 9-month-old by intraperitoneal injection. Vehicle group and its littermate wild-type mice were treated with the same volume of vehicles by intraperitoneal injection. NJK14047 was dissolved in pure DMSO at $25 \mathrm{mg} / \mathrm{ml}$ which was used for 40X stock solution. For working solution, the aliquot was diluted in PBS and filtered using $0.25 \mu \mathrm{m}$ syringe filter. The body weights of the mice did not significantly differ among groups and any conspicuous side effects (weight loss, anorexia, convulsion or death) were not observed. All mice used in this study were sacrificed at 9-month-old. Both sexes of mice were used in Morris water maze test and $A \beta_{1-42} E L S I A$ and there was no sex difference in therapeutic effects. To minimize the sex difference on the degree of amyloidopathy, the protein and RNA samples were acquired from male mice and histological sections were obtained from female mice. All experiments were approved by the Kyung Hee University Institutional Animal Care and Use Committee (IACUC, KHUASP(SE)-17-126-1).

Behavioral test. The Morris water maze task was used to assess spatial memory performance. The water maze was a white circular PVC tank (130-cm diameter, 40-cm height) in which water was filled to $31.5 \mathrm{~cm}$ in depth $\left(23 \pm 1^{\circ} \mathrm{C}\right)$. The submerged target platform was a $10 \mathrm{~cm}$ diameter circular zone $1.5 \mathrm{~cm}$ below the surface of the water. Titanium dioxide $\left(\mathrm{TiO}_{2}\right)$ was dispersed in the water to camouflage the target platform. The position of the platform was varied from mouse to mouse and distributed equally between experimental groups. One day before training, mice were habituated to the maze and swimming in the absence of cues. During the training period, mice were subjected to four trials per day. The start position of each trial was equally distributed. In each trial, the mouse was given $60 \mathrm{~s}$ to find the target platform in the presence of cues around the maze. The spent time before finding the platform was recorded as the latency of each trial. If the mouse did not find the platform within $60 \mathrm{~s}$, it was guided to the platform and allowed to stay on the platform for $10 \mathrm{~s}$. After a total 10 days of training, a probe test was performed (day 11). The mouse was subject to a trial in the absence of the platform for $60 \mathrm{~s}$. The start position was standardized to the opposite direction of the area in which the platform had existed. All trials were recorded using a camera and analyzed by a free tracking software tool, Toxtrac [28]. The software, the user manual, and the documentation are available at https://toxtrac.sourceforge.io. 
Immunoblotting. Immunoblotting analysis was performed as previously described [29]. In brief, mice were sacrificed after the behavioral test. Cortex and hippocampus were quickly isolated and stored at $-80^{\circ} \mathrm{C}$ until use. The brain tissues were homogenized in 10X volume of lysis buffer (RIPA buffer including $1 \%$ 100X protease inhibitor) and centrifuged at $13,000 \mathrm{rpm}$ for $20 \mathrm{~min}$. The supernatant was collected; concentrations were measured using the Bradford method (Bio-Rad, 5000006). Equal amounts of protein samples $(\sim 50 \mu \mathrm{g})$ were fractionated by SDS-PAGE, then transferred to PVDF membranes. The membranes were blocked with $5 \%$ skim milk and probed with primary antibodies at $4{ }^{\circ} \mathrm{C}$ overnight. After several washes in TBS-T, the membranes were probed with corresponding secondary antibodies conjugated with HRP. The immunoblot signals were developed with an enhanced chemiluminescence (ECL, Bio-Rad, 1705061) detection system. The band intensity was quantified using image $J$ densitometry $(\mathrm{NIH}$, Bethesda, MD, USA) and the data were normalized with respective $\beta$-actin level.

Tissue preparation and immunofluorescence. The mice were anesthetized with intraperitoneal injection of $2.5 \%$ Avertin (2, 2, 2-tribromoethanol) and immediately cardiac perfused with PBS followed by $4 \%$ paraformaldehyde in PBS. Brains were excised and post-fixed in $4 \%$ paraformaldehyde at $4{ }^{\circ} \mathrm{C}$ overnight and incubated in $30 \%$ sucrose at $4{ }^{\circ} \mathrm{C}$ until they had equilibrated. The brains were embedded into O.C.T. compound blocks at $-80^{\circ} \mathrm{C}$. Sequential $30-\mu \mathrm{m}$ coronal sections were obtained with a cryostat (CM30 50S; Leica). Every tenth section (300 $\mu \mathrm{m}$ apart) of the brain (Bregma -1.30 to $-2.70 \mathrm{~mm}$ ) was used for immunohistochemistry. Free-floating brain sections were rinsed in PBS, blocked for $1 \mathrm{~h}$ in $2 \%$ normal goat serum, $2 \%$ BSA and $0.4 \%$ Triton-X100, then incubated with primary antibodies at $4{ }^{\circ} \mathrm{C}$ overnight. After incubation with primary antibodies, sections were washed with PBS and incubated for $2 \mathrm{~h}$ with corresponding secondary antibodies conjugated with Alexa-Fluorescence.

Thioflavin S staining. To stain fibrillary $A \beta$, the brain sections were incubated with $0.1 \%$ Thioflavin $S$ in 50 $\% \mathrm{EtOH}$ for 10 min. Brain sections were sequentially washed with $50 \% \mathrm{EtOH}, 70 \% \mathrm{EtOH}, 100 \% \mathrm{EtOH}$ for 5 min respectively and coversliped with fluorescence mounting medium.

$\boldsymbol{A} \boldsymbol{\beta}$ ELISA. An enzyme linked immunosorbent assay (ELISA) for human $A \beta_{1-42}$ was performed using fluorescent-based ELISA kits (Invitrogen) and appropriate A $\beta$ standards, according to the manufacturer's protocol. The hippocampus and frontal cortex from one hemisphere were homogenized in 10X volume of guanidine buffer with a final concentration of $50 \mathrm{mM}$ Tris and $5 \mathrm{M}$ guanidine $\mathrm{HCl}, \mathrm{pH}$ 8.0. Homogenates were mixed at room temperature for 4 hours. After mixing, homogenates were diluted in PBS containing 5 $\%$ BSA, $0.03 \%$ Tween 20, and protease inhibitor cocktail.

Quantitative real time polymerase chain reaction (qRT-PCR). Total RNA extraction from mouse cortex and hippocampus was performed with a Hybrid-R total RNA purification kit (GeneAll ${ }^{\circledR}, 305-101$ ), according to the manufacturer's instructions. The concentration and purity of the RNA samples were assessed with a NanoDrop ${ }^{\text {TM }}$-2000c (ThermoFisher Scientific). cDNA synthesis was performed using TOPscript RT DryMIX (Enzynomics, RT200), according to the manufacturer's instructions. cDNA samples were subjected to qRTPCR using SYBR Green Mix (Enzynomics, RT500) and a CFX Connect real time PCR system (Bio-Rad). The qRT-PCR protocol was as follows: first holding stage at $95^{\circ} \mathrm{C}$ for $3 \mathrm{~min}$, followed by a cycling stage 
at $95^{\circ} \mathrm{C}$ for $10 \mathrm{~s}, 55^{\circ} \mathrm{C}$ for $10 \mathrm{~s}, 72{ }^{\circ} \mathrm{C}$ for $30 \mathrm{~s}\left(30\right.$ cycles total), and finally a holding stage at $95^{\circ} \mathrm{C}$ for 10 $\mathrm{s}$. The information about primers used in this study is listed in Table.2. The data were normalized with respective GAPDH level using $2^{-\triangle \triangle C T}$ method and shown as fold of control mean.

Fluoro-jade B staining. To stain late-stage degenerating neuronal cells, Fluoro-jade B staining was performed [30,31]. Brain sections were incubated with $1 \% \mathrm{NaOH}$ in $80 \% \mathrm{EtOH}$ for $5 \mathrm{~min}$, followed by sequential washes with $70 \% \mathrm{EtOH}$ and distilled water. Then the brain sections were incubated with 0.06 $\%$ potassium permanganate for $10 \mathrm{~min}$. After a 2-min wash with distilled water, the sections were incubated in $0.0004 \%$ Fluoro-jade B (Millipore, AG310) plus $0.01 \%$ acetic acid solution. After three washes with distilled water, the sections were fully dried and coverslipped with DPX mounting medium.

Cell lines and treatment. The BV2 (RRID: CVCL_0182) murine microglia cell line and the C8-D1A (RRID: CVCL_6379) murine astrocyte cell line were cultured in DMEM with $10 \% \mathrm{FBS}, 100 \mathrm{U} / \mathrm{ml}$ penicillin, and $100 \mu \mathrm{g} / \mathrm{ml}$ streptomycin at $37^{\circ} \mathrm{C}$ in a humidified atmosphere of $5 \% \mathrm{CO}_{2}$. Cells were seeded on 6-well plates at $510^{5}$ cells/well and stimulated with $500 \mathrm{ng} / \mathrm{ml}$ LPS after $2 \mathrm{~h}$ of pre-treatment with $1 \mu \mathrm{M}$ or 10 $\mu \mathrm{M}$ NJK14047. After $22 \mathrm{~h}$ of LPS stimulation, all medium was changed to fresh neurobasal medium. The cells were incubated in neurobasal medium for another $24 \mathrm{~h}$. The conditioned neurobasal medium was obtained and centrifuged at $1,500 \mathrm{rpm}$ for $5 \mathrm{~min}$ to remove the remaining cells. The supernatant was collected and stored at $4{ }^{\circ} \mathrm{C}$, then used to culture primary neurons within $24 \mathrm{~h}$.

Primary cells and treatment. Primary mouse cortical microglia and astrocytes were prepared according to public protocols [32, 33]. Briefly, mouse cortical mixed glia were obtained from P1 to P4 C57BL/6J mouse pups and seeded on poly-L-lysine pre-coated 75T flasks at $1.510^{7}$ cells/flask. Culture medium was changed every 3 days. After 5-7 days, to obtain microglia, the flasks were vigorously tapped and the supernatant which contained the microglia was collected and centrifuged at 3,000 rpm for $10 \mathrm{~min}$. The cell pellet was dispersed in new medium and seeded on poly-L-lysine pre-coated 6-well plates at $510^{5}$ cells/well. After $2 \mathrm{~h}$ of cell seeding, the culture medium was replaced with fresh medium to remove the oligodendrocytes. Approximately $96 \%$ of cells were found to be positive for a marker of microglia, ionized calcium-binding adapter molecule 1 (Iba-1). After flask tapping and supernatant discarding, to obtain astrocytes, the rest of the cells in the flask were rinsed with PBS and seeded onto poly-L-lysine pre-coated 6-well plates at $510^{5}$ cells/well. Approximately $98 \%$ of cells were positive for a marker of astrocytes, glial fibrillary acidic protein (GFAP). Prepared microglia and astrocytes were stimulated with $50 \mathrm{ng} / \mathrm{ml}$ LPS after $2 \mathrm{~h}$ of pre-treatment with $10 \mu \mathrm{M}$ NJK14047. After $22 \mathrm{~h}$ of LPS stimulation, all medium was changed to fresh neurobasal medium, in which the cells were incubated for another $24 \mathrm{~h}$. The conditioned neurobasal medium was obtained and centrifuged at 1,500 rpm for $5 \mathrm{~min}$. The supernatant was collected and stored at $4{ }^{\circ} \mathrm{C}$, then used to primary neurons within $24 \mathrm{~h}$.

Primary mice cortical neurons were prepared as previously described [34]. In brief, mouse cortical neurons were obtained from E17 C56BL/6J mouse embryos and seeded on poly-L-lysine pre-coated 48-well plates, for the MTT assay, or 24-well coverslips, for the TUNEL assay. Neurobasal medium including $2 \%$ B27 
supplement, 2 mM L-glutamine (Welgene, LS 002-01) and $1 \% \mathrm{P} / \mathrm{S}$ was used to culture neurons. Primary neurons were used in experiments at 10 days in vitro (DIV).

Cytotoxicity assay. MTT assay measures the metabolic activity of the cells so it is generally used to assess the cell viability [35]. To assess the cytotoxicity of glia-conditioned medium, mouse cortical primary neurons were seeded on poly-L-lysine pre-coated 48-well plates at $110^{5}$ cells/well. On DIV10, neurons were incubated in the BV2, C8-D1A, primary cultured microglia and primary cultured astrocyte conditioned medium for $24 \mathrm{~h}$. Media were exchanged for neurobasal media including $10 \%$ MTT. Absorbance at $570 \mathrm{~nm}$ corrected with $690 \mathrm{~nm}$ values were measured by a microplate reader. The data were normalized to the control group and shown as $\%$ of control mean.

Nitric oxide concentration measurement. To measure the Nitric oxide (NO) concentration in the conditioned medium obtained from each cell line, conditioned medium was mixed with Griess reagent at 1:1 ratio. Absorbance at $550 \mathrm{~nm}$ values were measured by a microplate reader.

TUNEL assay. TUNEL is a widely used method to detecting apoptotic DNA fragmentation [36]. To measure the apoptotic cell death of primary neuronal cells, mice cortical primary neurons were seeded on poly-L-lysine pre-coated 24-well cover glasses at $1.510^{5}$ cells/well. On DIV10, neurons were incubated with each conditioned medium for $24 \mathrm{~h}$. After incubation, the cells were subjected to TUNEL staining in according to the manufacturer's instructions. Apoptotic cells were detected as localized bright green cells (positive cells) in a blue background by using an Olympus BX51 microscope. The quantification of apoptosis was performed using Image $\mathrm{J}$ software.

Confocal microscopy and image analysis. All stained brain sections were imaged by confocal microscopy. Z-stacked images were acquired at $1.5 \mu \mathrm{m}$ intervals (total 15 optical slices). Four cortex area (2 left; 2 right) and four hippocampus area (2 left; 2 right) were imaged in one brain slice. Four to five brain slices (Bregma -1.30 to $-2.70 \mathrm{~mm}, 300 \mu \mathrm{m}$ apart) were used to quantify one mouse. Thioflavin $\mathrm{S}$ positive area, $6 \mathrm{E} 10$ positive area and Fluoro-jade B positive cells were quantified using Image $\mathrm{J}$ software. The number of Iba-1 positive cells and GFAP positive cells were quantified using Cell Profiler software [37]. To quantify the number of plaque associated microglia, Iba-1 positive cells within $20 \mu \mathrm{m}$ range of plaques were manually counted. Total $70-75$ plaques $(>10 \mu \mathrm{m})$ from five mouse per group were measured [26].

Statistical analysis. In all in vivo studies, the ' $n$ ' number means the number of animals used in the statistical analysis. Regarding in vitro studies, the ' $n$ ' number means the number of independent experiments. The group sizes for each experiment was based on our previous results [38]. The operators responsible for experimental procedure and data analysis were blinded and unaware of group allocation throughout the experiments. All data were analyzed using SPSS ver.25 (IBM corporation, NY, USA) and $P$ values less than 0.05 were considered statistically significant. Parametric tests were used when the data satisfied with the null hypothesis of Levene's test. Tukey's post-hoc test were used if the $p$-value $<0.05$ in one-way ANOVA. In case of qRT-PCR and MTT assay, Kruskal-Wallis test followed by Dunn's multiple 
comparison test was used. Latency of Morris water maze task was analyzed using generalized estimating equation (GEE) analysis. To analyze the group differences of each day, one-way ANOVA was used satisfying the assumption of Levene's test followed by Tukey's post-hoc test. The general data were expressed as mean \pm standard error of the mean (SEM) and all graphs were drawn using Graph Pad Prism 5.0 software (Graph Pad software Inc., CA, USA).

\section{Results}

\section{NJK14047, a selective p38a/ $\beta$ MAPKs inhibitor, reduces the level of phospho-p38 MAPKs in brain and attenuates the spatial memory loss in 9-month-old 5XFAD mice.}

The phospho-p38 MAPKs level was upregulated in the brain of 9-month-old 5XFAD mice in both regions of the brain, cortex and hippocampus, compared to the wild-type mice (Fig. 1A). Interestingly, the upregulated phospho-p38 MAPKs levels in the 5XFAD mice brain were significantly reduced in NJK14047 treated 5XFAD mice brain, and the levels were comparable to those of the WT mice. This inhibitory effect was shown in both cortex and hippocampus regions, respectively (Fig 1A).

Suppression of p38 MAPKs pathway in 5XFAD mice by NJK14047 prompted us to test whether NJK14047 treatment could ameliorate the spatial learning memory impairment of 5XFAD mice. NJK14047 treated and vehicle treated 5XFAD mice and their wild-type littermates were subjected to Morris water maze task [39]. As shown in Figure 1B, 5XFAD mice showed markedly longer escape latency than wild-type mice, and the gap between two groups was statistically significant since day 6 . However, NJK14047 treated 5XFAD mice showed decreasing escape latency, and the gap between

5XFAD+NJK14047 group and 5XFAD group became statistically significant since day 8 (Fig. 1B). At day 11 , the hidden platform was removed and a probe task was performed. Representative swimming paths of each group during the probe task were shown in Figure 1B. 5XFAD mice represented less total swimming distance as compared with wild-type littermates, but no significant difference between NJK14047 treated and vehicle treated groups (Fig. 1C). Spending time in target quadrant, wild-type mice recorded $24.48 \pm 2.14 \mathrm{~s}$, while 5XFAD mice recorded $13.14 \pm 2.35 \mathrm{~s}(P<0.01)$. The difference between wild-type and 5XFAD mice was diminished by NJK14047 treatment (23.79 $\pm 2.08 \mathrm{~s}, P<0.01)$ (Fig. 1D). Target zone cross also differed between groups. Wild-type group and 5XFAD+NJK14047 group showed $4.25 \pm 0.77$ times and $4.00 \pm 0.62$ times, respectively, whereas 5XFAD group had less times of the zone cross in a $60 \mathrm{~s}$ probe task $(1.50 \pm 0.57$ times, $P<0.05)$ (Fig. 1E). These results suggest that NJK14047 treatment reduces phohpo-p38 MAPK levels in 5XFAD mice brain and prevents spatial learning memory loss of 9-month-old 5XFAD mice.

\section{NJK14047 decreases A $\beta$ deposits in the brain of 9-month-old 5XFAD mice.}

As a next step, we investigated whether $A \beta$ deposits, one of the representative hallmarks in $A D$, were changed in NJK14047 treated 5XFAD mice. As shown in Figure 2A, thioflavin S positive A $\beta$ plaques were observed in 9-month-old 5XFAD mice, while no plaques were detected in their wild-type littermates. Notably, the thioflavin S positive area was decreased in both cortex and hippocampus regions of 
NJK14047 treated 5XFAD mice. Immunostaining using a 6E10 antibody against human $A \beta_{1-16}$ showed similar results, supporting the reduction of $A \beta$ plaques by NJK14047 treatment in the brain (Fig. 2B). This $A \beta$ reduction effect of NJK14047 was further confirmed using human $A \beta_{1-42}$ ELISA kit (Fig. 2C). These data reveal that NJK14047 treatment attenuate $A \beta$ deposits in 9-month-old 5XFAD mice brain.

\section{A $\beta$ processing and degradation are not affected by NJK14047.}

Reduction of Aß plaques in NJK14047 treated mice brain might result from the decrease of APP production or the reduced level of proteins related to $A \beta$ processing or degradation. To investigate this hypothesis, the protein levels of hAPP, BACE1, and PS1 were examined by immunoblotting. No significant change in hAPP, BACE1, and PS1 levels was observed in NJK14047 treated mice brains compared to those of 5XFAD mice, suggesting that the reduction of $A \beta$ plaque by NJK14047 does not result from the reduction of APP processing (Fig. $3 \mathrm{~A}$ ). In addition to APP processing molecules, proteins involved in A $\beta$ degradation, such as insulin degrading enzyme (IDE) and neutral endopeptidase (neprilysin, NEP), could also regulate the $A \beta$ deposits. $[40,41]$. Similar to APP processing molecules, the protein levels of $A \beta$ degrading enzymes did not show significant differences between the experimental groups (Fig. 3B). These results suggest that NJK14047 treatment could not directly change the APP processing pathway or $A \beta$ degrading enzymes in 9-month-old 5XFAD mice.

\section{NJK14047 inhibits neuroinflammatory conditions in the brain of 9-month-old 5XFAD mice.}

Because neuroinflammation is considered to contribute $A D$ pathologies and is related to $A \beta$ generation and clearance [6, 42], we examined the neuroinflammation state in our 5XFAD mice. First, we assessed the gliosis of microglia and astrocytes in 5XFAD mice. As expected, 9-month-old 5XFAD mice exhibited higher microgliosis rate than wild-type littermates in cortex and hippocampus. This upregulated microgliosis rate was significantly decreased in NJK1407 treated 5XFAD mice (Fig 4A). Similar to microgliosis, increased astrogliosis rate was also decreased in NJK1407 treated 5XFAD group (Fig 4B). To further investigate these, we tried to analyze the microglial activation markers using qRT-PCR. In accordance with the gliosis results, expression levels of pro-inflammatory cytokines such as tumor necrosis factor a (TNF- $\alpha$ ), interleukin $1 \beta$ (IL-1 $\beta)$, and interleukin 6 (IL-6) were significantly elevated in 5XFAD mice brain compared to those of wild-type mice (Fig. 4C, upper). On the other hand, arginase 1 (Arg1), chil3 chitinase-like 3 (Chi3/3/YM-1), and resistin like alpha (Retnla/Fizz-1) which indicate alternative active state of microglia were not different between wild-type and 5XFAD mice except Fizz-1 (Fig. 4C, lower). The expression levels of pro-inflammatory cytokines were significantly decreased in NJK1407 treated 5XFAD mice brain except TNF-a (Fig. 4C, upper). On the contrary, Arg1, YM-1 and Fizz-1 were upregulated in NJK14047 treated 5XFAD mice cortex. In hippocampus, Arg1 and YM-1 expression levels tended to be increased in 5XFAD+NJK14047 group and Fizz-1 expression level was significantly increased compared to 5XFAD group (Fig. 4C, lower). Taken together, these results indicate that the NJK14047 treatment alleviates inflammatory conditions in 9-month-old 5XFAD mice brain.

\section{NJK14047 increases phagocytic activity of microglia relating to $A \beta$ clearance.}


It has been demonstrated that the alternative activation of microglia was efficient for phagocytizing and degrading $A \beta$ peptides [43]. For further confirmation of alleviation of neuroinflammatory condition in 5XFAD mice by NJK14047 treatment, we investigated phagocytic function of microglia in the 9-month-old 5XFAD mice. The mRNA expression levels of phagocytic receptors in the brain; macrophage receptor with collagenous structure (MACRO), macrophage scavenger receptor 1 (Msr1), scavenger receptor class $B$, member 1 (Scarb1), and scavenger receptor class B, member 3 (Scarb3) were measured using qRT-PCR. As expected, NJK14047 treated 5XFAD mice showed increased expression levels of phagocytic receptors compared with wild-type mice or vehicle treated 5XFAD mice except Scarb1 (Fig. 5A). For histological examination, the brain sections were co-stained with thioflavin $S$ and Iba- 1 antibody. Consistent with qRTPCR data, we observed that microglia were often close to $A \beta$ plaque and surrounded it in NJK14047 treated mice brain (Fig. 5B). These data indicate that NJK14047 treatment induces anti-inflammatory conditions and improves microglial phagocytic function on $A \beta$ peptides in 9-month-old 5XFAD mice.

\section{NJK14047 reduces the neuronal cell death in cortex and hippocampus.}

It has been reported that the $A \beta$ deposits and chronic neuroinflammation may provoke neuronal cell death, which is one of the critical pathologies in $A D$ [44-47]. Since our data showed decrease of $A \beta$ plaques and neuroinflammation in NJK14047 treated 9-month-old 5XFAD mice, we speculated that the p38 MAPK inhibitor might reduce the neuronal cell death in 5XFAD mice and tried to confirm it. As expected, we observed Fluoro-jade $B$ positive cells in cortex and hippocampus of 9-month-old 5XFAD mice, not in wild-type mice. In 5XFAD+NJK14047 group, there were less Fluoro-jade B positive cells in both brain regions compared to 5XFAD group (Fig. 6). This data is consistent with our speculation that neuronal cell death could be decreased through the reduction in $A \beta$ plaques, and the alleviation of neuroinflammatory state in the brain of SXFAD mice, which might be contributed by the pharmacological inhibition of $p 38 a / \beta$ MAPKs.

\section{NJK14047 decreases the activated microglia-mediated neurotoxicity.}

To delineate the relations between suppression of neuroinflammation by NJK14047 and its neuroprotective effect in AD mice, we examined the effect of NJK14047 using primary neurons, microglial cell line BV2 and astrocyte cell line C8-D1A. BV2 and C8-D1A were cultured in vitro, and the conditioned media (CM) of BV2 and C8-D1A were prepared as described in "Materials and methods." Each CM was treated in mouse primary cortical neurons for $24 \mathrm{~h}$ and the cell viability was assessed (Fig 7A). As shown in Fig 7B, we observed neurotoxicity of LPS-stimulated BV2 CM. However, pre-treatment of NJK14047 in BV2 cells alleviated the neurotoxicity induced by LPS-stimulated BV2 CM (Fig. 7B, left). In contrast with LPS-stimulated BV2 CM, LPS-stimulated C8-D1A CM showed no neurotoxicity at all (Fig. 7B, right). To confirm the cell viability data, we proceeded TUNEL assay and found that TUNEL positive neurons were increased in LPS-stimulated BV2 CM treated group. While, as expected, TUNEL positive cells were decreased in NJK14047 treated BV2 CM group (Fig. 7C).

Given the observation that NJK14047 can decrease the neurotoxicity of LPS-stimulated BV2 CM, we intended to confirm these results using primary microglia and astrocytes. Primary microglia conditioned 
medium (MCM) and astrocytes conditioned medium (ACM) were prepared in accordance with the protocols in "Materials and methods." Primary neurons were incubated with each CM for $24 \mathrm{~h}$ and subjected to MTT and TUNEL assay (Fig. 8A). Similar to cell line study, LPS-stimulated MCM was shown neurotoxicity which was reversed by NJK14047 while ACM showed no differences (Fig. 8B). In consistent with MTT assay, there were more TUNEL positive cells in LPS-stimulated MCM group in comparison with naïve MCM group. This elevation was decreased in NJK14047 treated MCM group. ACM treated neurons showed no significant changes as expected (Fig 8C). Collectively, these findings demonstrate that reactive microglia, rather than astrocytes, provoke the apoptosis of neurons and NJK14047 can attenuate the neurotoxicity of LPS-stimulated MCM.

\section{Discussion And Conclusions}

5XFAD mouse is a transgenic AD mouse model which is characterized by a rapid $A \beta$ accumulation in the brain, leading to chronic neuroinflammation. This model exhibits $A \beta$ aggregation and gliosis from 2 months of age and neuronal loss from 6 months of age [24]. 5XFAD mice showed impaired spatial learning memory after 9 months of age [48], and motor function deficits after 12 months of age [49]. In present study, we showed that NJK14047 treatment from 6-month-old 5XFAD mice could significantly ameliorate $A \beta$-induced neuroinflammation leading to alleviation of neuronal degeneration and cognitive impairment at 9-month-old 5XFAD mice.

Numerous studies have attempted to attenuate $A \beta$-induced neurotoxicity and memory impairment by inhibiting p38 MAPKs signaling [50,51]. Most studies have been performed using induced neurodegenerative in vivo models or indirect p38 MAPKs inhibitors. One group has recently reported therapeutic effects in 11-12-month-old APP/PS1 mouse using the direct p38a MAPK inhibitor, MW150 [20, 52], and in 20-month-old hTau mouse using MW181 [53]. Therefore, p38 MAPK inhibition is a promising target for $A D$ therapy. In this study, we exhibited the therapeutic effects of a direct and selective p38a/ $\beta$ MAPKs inhibitor NJK14047 and investigated its mode of action in 9-month-old 5XFAD mice. P38 MAPKs are activated in chronic inflammatory diseases such as $A D$ and several studies have shown the up-regulation of phospho-p38 levels in the brain of AD mice models, but not in 5XFAD mice. We revealed that the phospho-p38 level was also upregulated in the cortex and hippocampus of 9-month-old 5XFAD mice and it could be reversed by NJK14047. Blood-brain barrier (BBB) is a grave obstruction for many brain target drugs, but NJK14047 successfully achieved its inhibitory effect on p38 MAPKs in cortex and hippocampus of 5XFAD mice. In fact, it is plausible that NJK14047 could show the effect because the BBB of old 5XFAD mice was collapsed due to chronic inflammatory conditions. Its low molecular weight $(\mathrm{MW}=445.515)$ and appropriate lipophilicity (cLogP $=3.12)$ also might contribute to the efficient delivery into brain. Further experiments are required to define whether NJK14047 can pass the intact BBB in normal conditions.

Down-regulation of phospho-p38 MAPKs level is related to the decreased neuroinflammation, which can reverse reactive gliosis and overexpression of pro-inflammatory cytokines such as TNF-a, IL-1 $\beta$, and IL-6. Recent study using p38a MAPK inhibitor MW150 in 11-12-month-old APP/PS1 mice showed decreased 
IL-1 $\beta$ and TNF-a levels and increased number of microglial cells around the plaque [20]. Similar to previous report, we confirmed that NJK14047, a selective $p 38 \alpha / \beta$ inhibitor, also decreased the proinflammatory cytokines and upregulated alternative activated microglial markers such as Arg1, YM-1, and Fizz-1 in cortex and hippocampus of 5XFAD mice. In addition, NJK14047 treatment increased the mRNA expression of microglial phagocytic receptors in the brain and improved the number of plaque associated microglia, suggesting the recovery of microglial phagocytic activity [54]. In neuroinflammation conditions, resident microglia or infiltrated macrophages were able to polarize into pro-inflammatory phenotype (M1) upon exposure to pro-inflammatory cytokines or protein debris. These cells express high level of proinflammatory cytokines and reactive oxygen species which can be toxic to neurons. On the contrary, it has been recognized that alternative activation of microglia (M2) has positive influences on tissue repair, immunological regulation, and phagocytosis-mediated $A \beta$ clearance $[43,55]$. Thus, there is a possibility that NJK14047 could change the ratio of microglial population from M1 phenotype into M2 phenotype and lead to ameliorate AD pathology. However, this dichotomous nomenclature of in vivo microglia may be an oversimplified classification [56]. More specific and detailed analysis and classifications are required to evaluate the microglial variation by NJK14047 treatment.

$A \beta$ peptide was generated from APP, which could be processed by $\beta$-secretase and $\gamma$-secretase complex, respectively, and accumulated in the extracellular matrix in $A D$. A $\beta$ peptides could be degraded by proteolytic enzymes such as IDE, NEP, and MMP-9 or phagocytosed by microglia or astrocytes. Our study demonstrates that NJK14047 treatment reduced the A $\beta$ accumulation in 9-month-old 5XFAD mice. But we observed no significant differences in APP processing enzymes or A $\beta$ degradation enzymes between the groups. Recent studies about p38a MAPK and AD demonstrated that genetic modulation of neuronal p38a MAPK could ameliorate AD pathologies in AD mouse models $[57,58]$. They showed that downregulation of p38a MAPK in neurons reduced BACE1 activity thereby lowering $A \beta$ accumulation in early stage AD mice. Interestingly, Colié et al. revealed that there were no significant differences of BACE1 protein levels between the experimental groups in 12-month-old 5XFAD mice, indicating that the effect of neuronal p38a inhibition to BACE1 levels could be different depending on the age of 5XFAD mice [57]. Based on these results, NJK14047 might inhibit the neuronal p38a/ $\beta$ MAPKs and decrease the BACE1 levels in the early phase of treatment, but this effect probably be diminished in 9-month-old mice at which we analyzed. Instead of the decreased BACE1 levels, we found that NJK14047 treatment ameliorated neuroinflammatory conditions and increased phagocytic receptors of microglia and the number of microglia around the $A \beta$ plaque. These enhanced microglial phagocytic function might also contribute to decrease $A \beta$ accumulation.

Another possible cause of $A \beta$ reduction and restoration of microglial phagocytic function is the effect of $p 38 \beta$ MAPK inhibition of NJK14047. According to the previous study, both $p 38 a$ and $p 38 \beta$ MAPKs were highly active in basal brain condition. However, $p 38 a$ and p38 $\beta$ showed different localization, indicating that they might have distinct roles or mechanisms. Especially, p38 $\beta$ stained in glial cells in many brain regions as well as neuronal nuclei [16]. Thus, p38 $\beta$ MAPK inhibition might be important to ameliorate the inflammatory conditions of glial cells successfully and it could be suggested that both $p 38 \alpha$ and $p 38 \beta$ 
dual inhibition is efficient therapeutic strategy to treat AD. Further studies are required to clarify this point of view.

At a final stage of $A D$, irreversible neuronal loss is found at the cortex and hippocampus which are contributed in encoding of new information and consolidation of memory networks. In that point, protection of neurons against harmful enemies such as $A \beta$ plaques or massive pro-inflammatory signals is important for AD therapy. We found that NJK14047 treated 5XFAD mice exhibited improved spatial learning memory than vehicle treated 5XFAD mice. Moreover, we ascertained that neuronal degeneration was significantly decreased in the brain of NJK14047 treated mice. We also confirmed neuroprotective effect of NJK14047 in in vitro studies. The neurons were under apoptosis in LPS-stimulated BV2 CM but NJK14047 treatment to BV2 cells reversed this result. Interestingly, LPS-stimulated C8-D1A CM had not shown any toxicity to neurons. The anti-neurotoxic effect of NJK14047 was further confirmed using primary microglia and astrocytes. Consistent with cell line studies, LPS-stimulated MCM made neurons under apoptosis while NJK14047 pre-treatment to microglia alleviated the neurotoxicity. Similar to C8D1A, LPS-stimulated astrocytes were also little toxic to neurons. Recent studies have revealed that reactive astrocytes with $A 1$ phenotype are toxic to neurons. However, polarization into $A 1$ astrocytes needs the activated MCM especially including IL-1a, TNF, and C1q [59]. Collectively, it can be suggested that LPS-stimulated astrocytes without pro-inflammatory microglial activation are less toxic to neurons and that NJK14047 treatment inhibits the microglial activation and ultimately results in neuroprotection.

In summary, we have shown that pharmacological inhibition of $p 38 \alpha / \beta$ MAPKs in the brain of 5XFAD mice contributes to reduction of $A \beta$ accumulation and amelioration of neuroinflammatory conditions and microglial phagocytic functions which may contribute to decreased neuronal death and improved learning memory. However NJK14047 inhibits both p38a and p38 $\beta$ MAPKs and affects neurons and glia generally. It should be needed further studies by which p38 MAPKs related to the therapeutic effects of NJK14047 in neurons and in glia. Taken together, a selective p38a/ $\beta$ MAPKs inhibitor NJK14047 successfully showed therapeutic effects in 5XFAD mice. Our data support that p38 MAPKs inhibition is a potential strategy for AD therapy and NJK14047 might be one of the promising candidates for AD therapeutics targeting p38 MAPKs.

\section{Abbreviations}

$A \beta$, amyloid beta; $A C M$, astrocyte conditioned medium; $A D$, Alzheimer's disease; Arg1, arginase 1; BACE1, beta-site APP cleaving enzyme 1; BBB, blood-brain barrier; Chi3l3/YM-1, chil3 chitinase-like 3; CM, conditioned medium; GFAP, glial fibrillary acidic protein; hAPP, human amyloid precursor protein; Iba-1, ionized calcium-binding adapter molecule 1; IDE, insulin degrading enzyme; MACRO, macrophage receptor with collagenous structure; $\mathrm{MCM}$, microglia conditioned medium; Msr1, macrophage scavenger receptor 1; NEP, neprilysin; NO, nitric oxide; PS1, presenilin 1; Retnla/Fizz-1, resistin like alpha; Scarb1, scavenger receptor class $B$, member 1 ; Scarb3, scavenger receptor class $B$, member 3

\section{Declarations}




\section{Ethics approval and consent to participate}

All animal experiments were carried out in compliance with the Guide for the Care and Use of Laboratory Animals of the National Institutes of Health and were approved by the Kyung Hee University Institutional Animal Care and Use Committee (IACUC, KHUASP(SE)-17-126-1).

\section{Consent for publication}

Not applicable

\section{Availability of data and materials}

The datasets used and/or analyzed during the current study are available from the corresponding author on reasonable request.

\section{Competing Interests}

The authors declare no competing interest.

\section{Funding}

This research was supported by the Basic Science Research Program through the National Research Foundation of Korea (NRF) funded by the Ministry of Education (NRF-2018R1D1A1B07050547). This research was also supported by Basic Science Research Program through the National Research Foundation of Korea (NRF), which was funded by the Ministry of Science, ICT \& Future Planning (NRF2017R1A5A2014768).

\section{Author Contributions}

M.S.G and S.H.S performed the experiments, analyzed the data and prepared the manuscript. S.H.J and Y.J.J performed animal study. J.D and N.K provided mouse primary cells. S.J.L synthesized and provided NJK14047. K.S.I, N.J.K and J.K.L interpreted the data and reviewed the manuscript. E.K.C performed statistical analysis of the data. K.S.I, N.J.K and J.K.L. designed the study and wrote the manuscript. All authors discussed results and commented on the manuscript.

\section{Acknowledgements}

Not applicable

\section{References}

1. Huang Y, Mucke L: Alzheimer mechanisms and therapeutic strategies. Cell 2012, 148(6):1204-1222.

2. Gouras GK, Almeida CG, Takahashi RH: Intraneuronal Abeta accumulation and origin of plaques in Alzheimer's disease. Neurobiology of aging 2005, 26(9):1235-1244. 
3. Yiannopoulou KG, Papageorgiou SG: Current and future treatments for Alzheimer's disease. Therapeutic advances in neurological disorders 2013, 6(1):19-33.

4. Murphy MP, LeVine H, 3rd: Alzheimer's disease and the amyloid-beta peptide. Journal of Alzheimer's disease : JAD 2010, 19(1):311-323.

5. Yang TT, Hsu CT, Kuo YM: Cell-derived soluble oligomers of human amyloid-beta peptides disturb cellular homeostasis and induce apoptosis in primary hippocampal neurons. Journal of neural transmission (Vienna, Austria : 1996) 2009, 116(12):1561-1569.

6. Cai Z, Hussain MD, Yan LJ: Microglia, neuroinflammation, and beta-amyloid protein in Alzheimer's disease. The International journal of neuroscience 2014, 124(5):307-321.

7. Ardura-Fabregat A, Boddeke E, Boza-Serrano A, Brioschi S, Castro-Gomez S, Ceyzeriat K, Dansokho C, Dierkes T, Gelders G, Heneka MT et al: Targeting Neuroinflammation to Treat Alzheimer's Disease. CNS drugs 2017, 31(12):1057-1082.

8. McManus RM, Heneka MT: Role of neuroinflammation in neurodegeneration: new insights. Alzheimer's research \& therapy 2017, 9(1):14.

9. Kim YS, Joh TH: Microglia, major player in the brain inflammation: their roles in the pathogenesis of Parkinson's disease. Experimental \& molecular medicine 2006, 38(4):333-347.

10. Clayton KA, Van Enoo AA, Ikezu T: Alzheimer's Disease: The Role of Microglia in Brain Homeostasis and Proteopathy. Frontiers in neuroscience 2017, 11:680.

11. Hansen DV, Hanson JE, Sheng M: Microglia in Alzheimer's disease. The Journal of cell biology 2018, 217(2):459-472.

12. Kaminska B, Gozdz A, Zawadzka M, Ellert-Miklaszewska A, Lipko M: MAPK signal transduction underlying brain inflammation and gliosis as therapeutic target. Anatomical record (Hoboken, NJ : 2007) 2009, 292(12):1902-1913.

13. Pearson G, Robinson F, Beers Gibson T, Xu BE, Karandikar M, Berman K, Cobb MH: Mitogen-activated protein (MAP) kinase pathways: regulation and physiological functions. Endocrine reviews 2001, 22(2):153-183.

14. Johnson GL, Lapadat R: Mitogen-activated protein kinase pathways mediated by ERK, JNK, and p38 protein kinases. Science (New York, NY) 2002, 298(5600):1911-1912.

15. Cuenda A, Rousseau S: p38 MAP-kinases pathway regulation, function and role in human diseases. Biochimica et biophysica acta 2007, 1773(8):1358-1375.

16. Lee SH, Park J, Che Y, Han PL, Lee JK: Constitutive activity and differential localization of p38alpha and p38beta MAPKs in adult mouse brain. Journal of neuroscience research 2000, 60(5):623-631.

17. Zhao YW, Pan YQ, Tang MM, Lin WJ: Blocking p38 Signaling Reduces the Activation of Proinflammatory Cytokines and the Phosphorylation of p38 in the Habenula and Reverses DepressiveLike Behaviors Induced by Neuroinflammation. Frontiers in pharmacology 2018, 9:511.

18. Yao Y, Huang JZ, Chen L, Chen Y, Li X: In vivo and in vitro studies on the roles of p38 mitogenactivated protein kinase and NADPH-cytochrome P450 reductase in Alzheimer's disease. 
Experimental and therapeutic medicine 2017, 14(5):4755-4760.

19. Alam JJ: Selective Brain-Targeted Antagonism of p38 MAPKalpha Reduces Hippocampal IL-1beta Levels and Improves Morris Water Maze Performance in Aged Rats. Journal of Alzheimer's disease : JAD 2015, 48(1):219-227.

20. Zhou Z, Bachstetter AD, Spani CB, Roy SM, Watterson DM, Van Eldik LJ: Retention of normal glia function by an isoform-selective protein kinase inhibitor drug candidate that modulates cytokine production and cognitive outcomes. Journal of neuroinflammation 2017, 14(1):75.

21. Alam J, Blackburn K, Patrick D: Neflamapimod: Clinical Phase 2b-Ready Oral Small Molecule Inhibitor of p38alpha to Reverse Synaptic Dysfunction in Early Alzheimer's Disease. The journal of prevention of Alzheimer's disease 2017, 4(4):273-278.

22. Scheltens P, Prins N, Lammertsma A, Yaqub M, Gouw A, Wink AM, Chu HM, van Berckel BNM, Alam J: An exploratory clinical study of p38alpha kinase inhibition in Alzheimer's disease. Annals of clinical and translational neurology 2018, 5(4):464-473.

23. Gee MS, Kim SW, Kim N, Lee SJ, Oh MS, Jin HK, Bae JS, Inn KS, Kim NJ, Lee JK: A Novel and Selective p38 Mitogen-Activated Protein Kinase Inhibitor Attenuates LPS-Induced Neuroinflammation in BV2 Microglia and a Mouse Model. Neurochemical research 2018, 43(12):2362-2371.

24. Oakley H, Cole SL, Logan S, Maus E, Shao P, Craft J, Guillozet-Bongaarts A, Ohno M, Disterhoft J, Van Eldik $L$ et al: Intraneuronal beta-amyloid aggregates, neurodegeneration, and neuron loss in transgenic mice with five familial Alzheimer's disease mutations: potential factors in amyloid plaque formation. The Journal of neuroscience : the official journal of the Society for Neuroscience 2006, 26(40):10129-10140.

25. Heo J, Shin H, Lee J, Kim T, Inn KS, Kim NJ: Synthesis and biological evaluation of Ncyclopropylbenzamide-benzophenone hybrids as novel and selective p38 mitogen activated protein kinase (MAPK) inhibitors. Bioorganic \& medicinal chemistry letters 2015, 25(17):3694-3698.

26. Pan RY, Ma J, Kong XX, Wang XF, Li SS, Qi XL, Yan YH, Cheng J, Liu Q, Jin W et al: Sodium rutin ameliorates Alzheimer's disease-like pathology by enhancing microglial amyloid-beta clearance. Science advances 2019, 5(2):eaau6328.

27. An J, Zhou Y, Zhang M, Xie Y, Ke S, Liu L, Pan X, Chen Z: Exenatide alleviates mitochondrial dysfunction and cognitive impairment in the 5XFAD mouse model of Alzheimer's disease. Behavioural brain research 2019, 370:111932.

28. Rodriguez A, Zhang H, Klaminder J, Brodin T, Andersson M: Toxld: an efficient algorithm to solve occlusions when tracking multiple animals. Sci Rep 2017, 7(1):14774.

29. Lee JK, Jin HK, Endo S, Schuchman EH, Carter JE, Bae JS: Intracerebral transplantation of bone marrow-derived mesenchymal stem cells reduces amyloid-beta deposition and rescues memory deficits in Alzheimer's disease mice by modulation of immune responses. Stem cells (Dayton, Ohio) 2010, 28(2):329-343.

30. Schmued LC, Hopkins KJ: Fluoro-Jade B: a high affinity fluorescent marker for the localization of neuronal degeneration. Brain research 2000, 874(2):123-130. 
31. Poirier JL, Capek R, De Koninck Y: Differential progression of Dark Neuron and Fluoro-Jade labelling in the rat hippocampus following pilocarpine-induced status epilepticus. Neuroscience 2000, 97(1):59-68.

32. Tamashiro TT, Dalgard CL, Byrnes KR: Primary microglia isolation from mixed glial cell cultures of neonatal rat brain tissue. Journal of visualized experiments : JoVE 2012(66):e3814.

33. Lian H, Roy E, Zheng H: Protocol for Primary Microglial Culture Preparation. Bio-protocol 2016, 6(21).

34. Lee JK, Jin HK, Bae JS: Bone marrow-derived mesenchymal stem cells attenuate amyloid betainduced memory impairment and apoptosis by inhibiting neuronal cell death. Current Alzheimer research 2010, 7(6):540-548.

35. Stockert JC, Horobin RW, Colombo LL, Blazquez-Castro A: Tetrazolium salts and formazan products in Cell Biology: Viability assessment, fluorescence imaging, and labeling perspectives. Acta histochemica 2018, 120(3):159-167.

36. Gorczyca W, Bruno S, Darzynkiewicz R, Gong J, Darzynkiewicz Z: DNA strand breaks occurring during apoptosis - their early insitu detection by the terminal deoxynucleotidyl transferase and nick translation assays and prevention by serine protease inhibitors. International journal of oncology 1992, 1(6):639-648.

37. Carpenter AE, Jones TR, Lamprecht MR, Clarke C, Kang IH, Friman O, Guertin DA, Chang JH, Lindquist RA, Moffat $\mathrm{J}$ et al: CellProfiler: image analysis software for identifying and quantifying cell phenotypes. Genome Biology 2006, 7(10):R100.

38. Kim N, Do J, Ju IG, Jeon SH, Lee JK, Oh MS: Picrorhiza kurroa Prevents Memory Deficits by Inhibiting NLRP3 Inflammasome Activation and BACE1 Expression in 5xFAD Mice. Neurotherapeutics : the journal of the American Society for Experimental NeuroTherapeutics 2019.

39. Vorhees CV, Williams MT: Morris water maze: procedures for assessing spatial and related forms of learning and memory. Nature protocols 2006, 1(2):848-858.

40. Kurochkin IV, Goto S: Alzheimer's beta-amyloid peptide specifically interacts with and is degraded by insulin degrading enzyme. FEBS letters 1994, 345(1):33-37.

41. Iwata N, Tsubuki S, Takaki Y, Watanabe K, Sekiguchi M, Hosoki E, Kawashima-Morishima M, Lee HJ, Hama E, Sekine-Aizawa $Y$ et al: Identification of the major Abeta1-42-degrading catabolic pathway in brain parenchyma: suppression leads to biochemical and pathological deposition. Nature medicine 2000, 6(2):143-150.

42. Heneka MT, Carson MJ, El Khoury J, Landreth GE, Brosseron F, Feinstein DL, Jacobs AH, Wyss-Coray T, Vitorica J, Ransohoff RM et al: Neuroinflammation in Alzheimer's disease. The Lancet Neurology 2015, 14(4):388-405.

43. Cherry JD, Olschowka JA, O'Banion MK: Neuroinflammation and M2 microglia: the good, the bad, and the inflamed. Journal of neuroinflammation 2014, 11:98.

44. Chen WW, Zhang X, Huang WJ: Role of neuroinflammation in neurodegenerative diseases (Review). Molecular medicine reports 2016, 13(4):3391-3396. 
45. Skaper SD, Facci L, Zusso M, Giusti P: An Inflammation-Centric View of Neurological Disease: Beyond the Neuron. Frontiers in cellular neuroscience 2018, 12:72.

46. Bayer TA, Wirths O: Intracellular accumulation of amyloid-Beta - a predictor for synaptic dysfunction and neuron loss in Alzheimer's disease. Frontiers in aging neuroscience 2010, 2:8.

47. Morishima Y, Gotoh Y, Zieg J, Barrett T, Takano H, Flavell R, Davis RJ, Shirasaki Y, Greenberg ME: $\boldsymbol{\beta}$ Amyloid Induces Neuronal Apoptosis Via a Mechanism that Involves the c-Jun N-Terminal Kinase Pathway and the Induction of Fas Ligand. The Journal of Neuroscience 2001, 21(19):7551-7560.

48. Schneider F, Baldauf K, Wetzel W, Reymann KG: Behavioral and EEG changes in male 5xFAD mice. Physiology \& behavior 2014, 135:25-33.

49. O'Leary TP, Robertson A, Chipman PH, Rafuse VF, Brown RE: Motor function deficits in the 12 monthold female 5XFAD mouse model of Alzheimer's disease. Behavioural brain research 2018, 337:256263.

50. Lee JK, Kim NJ: Recent Advances in the Inhibition of p38 MAPK as a Potential Strategy for the Treatment of Alzheimer's Disease. Molecules (Basel, Switzerland) 2017, 22(8).

51. Kheiri G, Dolatshahi M, Rahmani F, Rezaei N: Role of p38/MAPKs in Alzheimer's disease: implications for amyloid beta toxicity targeted therapy. Reviews in the neurosciences 2018, 30(1):9-30.

52. Roy SM, Grum-Tokars VL, Schavocky JP, Saeed F, Staniszewski A, Teich AF, Arancio O, Bachstetter AD, Webster SJ, Van Eldik LJ et al: Targeting human central nervous system protein kinases: An isoform selective p38alphaMAPK inhibitor that attenuates disease progression in Alzheimer's disease mouse models. ACS chemical neuroscience 2015, 6(4):666-680.

53. Maphis N, Jiang S, Xu G, Kokiko-Cochran ON, Roy SM, Van Eldik LJ, Watterson DM, Lamb BT, Bhaskar K: Selective suppression of the alpha isoform of p38 MAPK rescues late-stage tau pathology. Alzheimer's research \& therapy 2016, 8(1):54.

54. Wilkinson K, El Khoury J: Microglial scavenger receptors and their roles in the pathogenesis of Alzheimer's disease. International journal of Alzheimer's disease 2012, 2012:489456.

55. Orihuela R, McPherson CA, Harry GJ: Microglial M1/M2 polarization and metabolic states. British journal of pharmacology 2016, 173(4):649-665.

56. Ransohoff RM: A polarizing question: do M1 and M2 microglia exist? Nature neuroscience 2016, 19(8):987-991.

57. Colie S, Sarroca S, Palenzuela R, Garcia I, Matheu A, Corpas R, Dotti CG, Esteban JA, Sanfeliu C, Nebreda AR: Neuronal p38alpha mediates synaptic and cognitive dysfunction in an Alzheimer's mouse model by controlling beta-amyloid production. Scientific reports 2017, 7:45306.

58. Schnoder L, Hao W, Qin Y, Liu S, Tomic I, Liu X, Fassbender K, Liu Y: Deficiency of Neuronal p38alpha MAPK Attenuates Amyloid Pathology in Alzheimer Disease Mouse and Cell Models through Facilitating Lysosomal Degradation of BACE1. The Journal of biological chemistry 2016, 291(5):2067-2079.

59. Liddelow SA, Guttenplan KA, Clarke LE, Bennett FC, Bohlen CJ, Schirmer L, Bennett ML, Munch AE, Chung WS, Peterson TC et al: Neurotoxic reactive astrocytes are induced by activated microglia. 
Nature 2017, 541(7638):481-487.

\section{Tables}

Table.1 Information of immunostaining antibodies used in this study

\begin{tabular}{llllll}
\hline Target & Host & Source & Catalog no. & RRID & Application \\
\hline $\begin{array}{l}\text { phospho-p38 MAPK } \\
\text { p38 MAPK }\end{array}$ & Rabbit & Cell Signaling Technology & 9215 & AB_331762 & WB / 1:1000 \\
$\begin{array}{l}\text { Human } \beta \text {-amyloid 1-16 } \\
\text { (clone: 6E10) }\end{array}$ & Mousbit & Cell Signaling Technology & 9212 & AB_330713 & WB / 1:1000 \\
\hline BACE1 & & BioLegend & 803001 & AB_2564653 & WB / 1:1000 \\
Presenilin-1 & Mouse & Millipore & MAB5308 & AB_11212616 & WB / 1:1000 \\
\hline IDE & Rabbit & Cell Signaling Technology & 5643 & AB_10706356 & WB / 1:1000 \\
\hline NEP & Rabbit & Abcam & ab32216 & AB_775686 & WB / 1:1000 \\
\hline Iba-1 & Goat & R\&D System & AF1126 & AB_2144426 & WB / 1:1000 \\
\hline GFAP & Rabbit & Wako & 019-19741 & AB_839504 & IHC / 1:500 \\
\hline Lipocalin-2 & Chicken & Abcam & ab4674 & AB_304558 & IHC / 1:500 \\
\hline Rabbit & Abcam & ab63929 & AB_1140965 & WB / 1:1000 \\
\hline -actin (HRP) & Mouse & Santa Cruz Biotechnology & sc-47778 HRP & AB_2714189 & WB / 1:5000 \\
\hline Mouse IgG (HRP) & Goat & Santa Cruz Biotechnology & sc-2005 & AB_631736 & WB / 1:5000 \\
\hline Rabbit IgG (HRP) & Goat & Santa Cruz Biotechnology & sc-2054 & AB_631748 & WB / 1:5000 \\
\hline Goat IgG (HRP) & Donkey & Santa Cruz Biotechnology & sc-2020 & AB_631728 & WB / 1:5000 \\
\hline Mouse IgG (488) & Goat & Invitrogen & A11001 & AB_2534069 & IHC / 1:1000 \\
\hline Rabbit IgG (488) & Goat & Invitrogen & A11008 & AB_143165 & IHC / 1:1000 \\
\hline Rabbit IgG (594) & Goat & Invitrogen & A11012 & AB_2534079 & IHC / 1:1000 \\
\hline Chicken IgY (488) & Goat & Invitrogen & A11039 & AB_2534096 & IHC / 1:1000 \\
\hline
\end{tabular}

WB, Western blot; IHC, Immunohistochemistry.

\section{Table.2 Information of qRT-PCR primers used in this study}

\begin{tabular}{llll}
\hline Gene name & NCBI reference & Forward primer $\left(5^{\prime} \rightarrow 3^{\prime}\right)$ & Reverse primer $\left(5^{\prime} \rightarrow 3^{\prime}\right)$ \\
\hline TNF- $\alpha$ & NM_013693 & GATTATGGCTCAGGGTCCAA & GCTCCAGTGAATCGGAAAG \\
IL-1 $\beta$ & NM_008361 & CCCAAGCAATACCCAAAGAA & GCTTGTGCTCTGCTTGTGAG \\
\hline IL-6 & NM_031168 & CCGGAGAGGAGACTTCACAG & TTGCCATTGCACAACTCTTT \\
\hline Arg1 & NM_007482 & AGTTGGGTTCACTTCCATGA & CGATTCACCTGAGCTTTGAT \\
\hline YM-1 & NM_009892 & AGAGCAAGAAACAAGCATGG & CTGTACCAGCTGGGAAGAAA \\
\hline Fizz & NM_020509 & TCCAGCTAACTATCCCTCCACTGT & GGCCCATCTGTTCATAGT \\
MACRO & NM_010766 & CTGTGGCAATGGATCACTAGC & CTCCTGGCTGGTATGGACC \\
\hline Msr1 & NM_031195 & TGAACGAGAGGATGCTGACTG & GGAGGGGCCATTTTAGTGC \\
\hline SCARB1 & NM_016741 & TTTGGAGTGGTAGTAAAAAGGGC & TGACATCAGGGACTCAGAGTAG \\
\hline SCARB3 & NM_007643 & GAACCACTGCTTTCAAAAACTGG & TGCTGTTCTTTGCCACGTCA \\
\hline GAPDH & NM_008084 & TGAATACGGCTACAGCAACA & AGGCCCCTCCTGTTATTATG \\
\hline
\end{tabular}




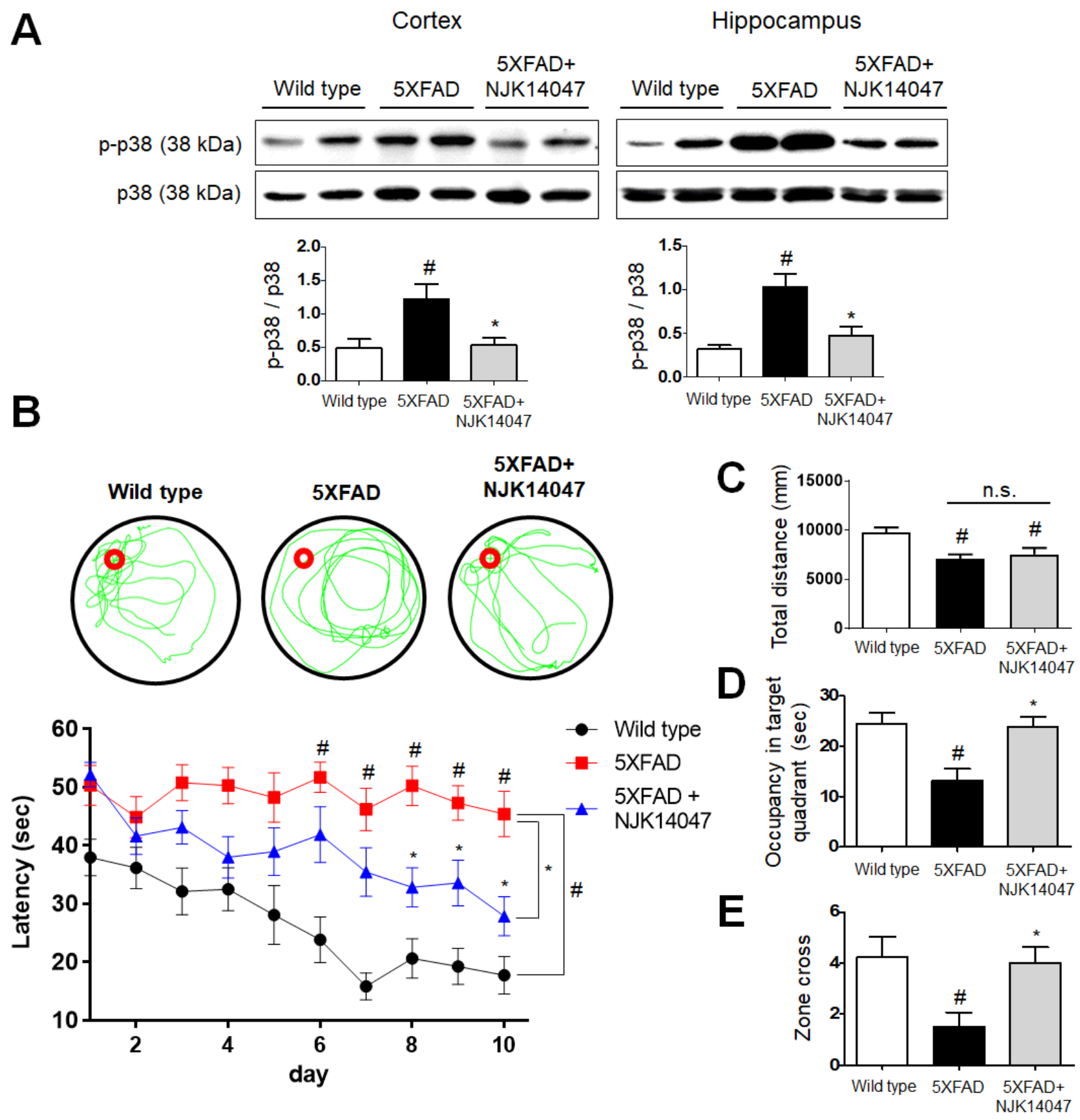

\section{Figure 1}

NJK14047 treatment from 6-month-old to 9-month-old reduces phospho-p38 MAPK level and prevents spatial learning memory impairment. (A) Inhibition of p38 MAPK in cortex and hippocampus of NJK14047 treated 9-month-old 5XFAD mice. Phospho-p38 protein level was normalized to total p38 level 
and shown as mean \pm SEM ( $\mathrm{n}=6$ per group, One-way ANOVA analysis). (B) Morris water maze task between three groups; wild-type, 5XFAD and 5XFAD+NJK14047. Representative swimming paths during a probe task after 10 days of training and escape latencies of each group for total 10 days of training. Data are shown as mean \pm SEM ( $n=12-13$ per group, GEE analysis). (C-E) After 10 days of training, a probe task was performed. (C) Total swimming distances were measured and there was no difference between 5XFAD and 5XFAD+NJK14047 group. (D) Times that the mice spent in the target quadrant and (E) the number of how many times the mice crossed the target zone during a 60-second probe test. Data are shown as mean \pm SEM ( $n=12-13$ per group, One-way ANOVA analysis). (\#P $<0.05$ vs. wild type; ${ }^{*}<0.05$ vs. 5XFAD)

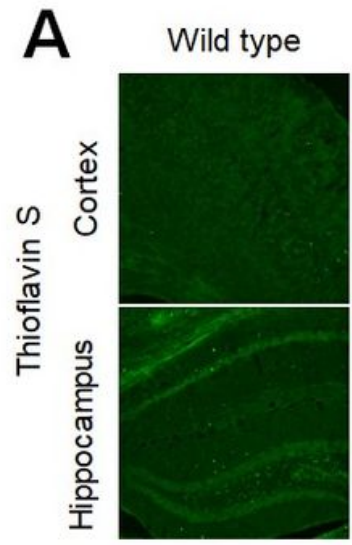

Cortex

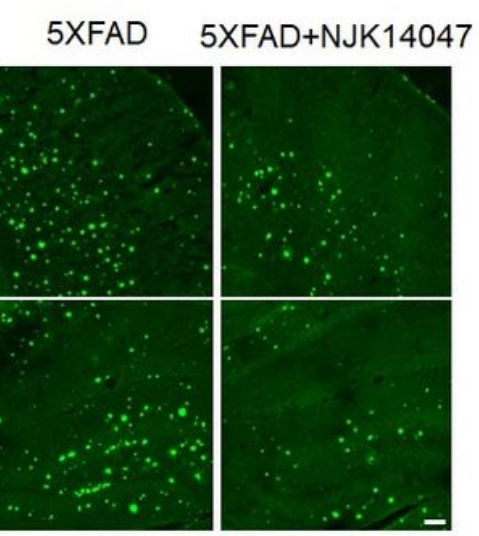

Hippocampus
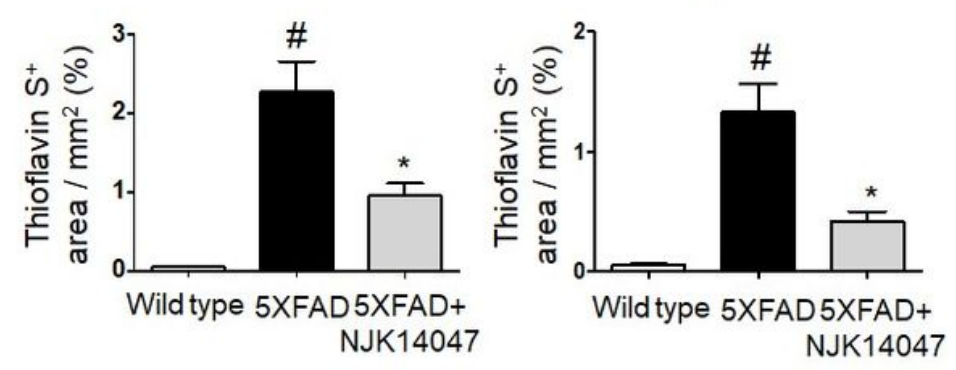
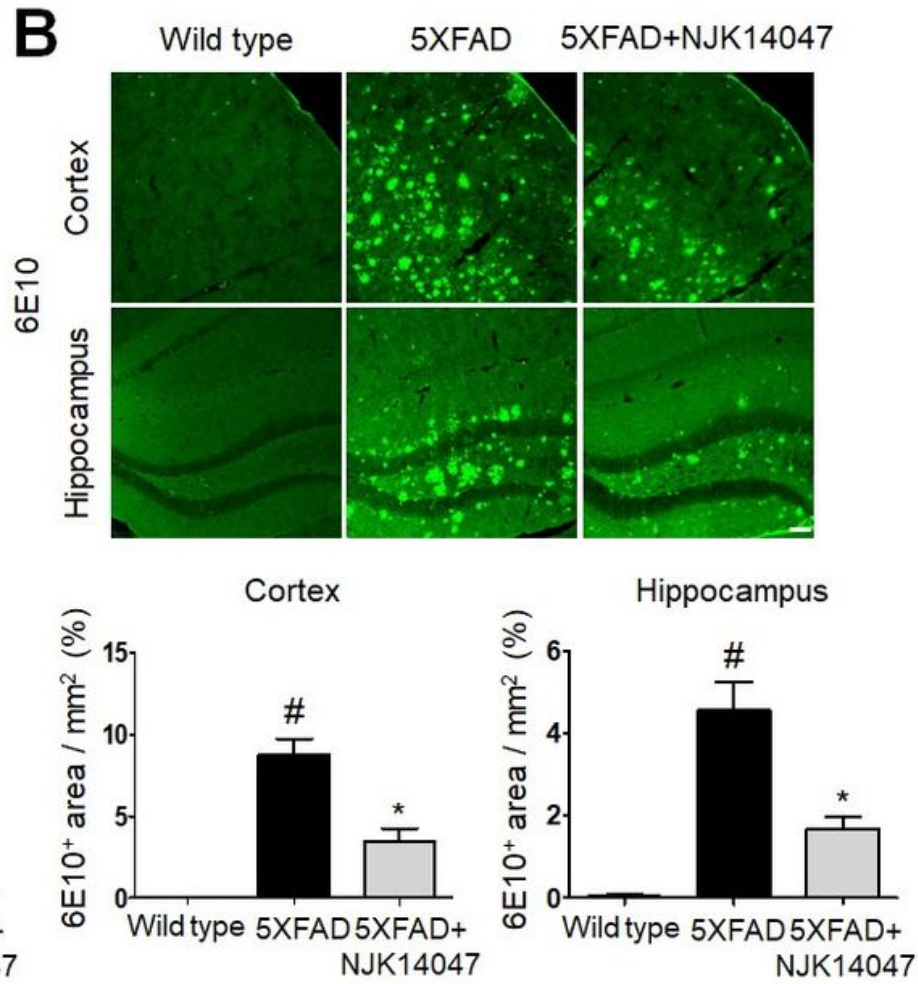

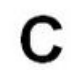

Cortex

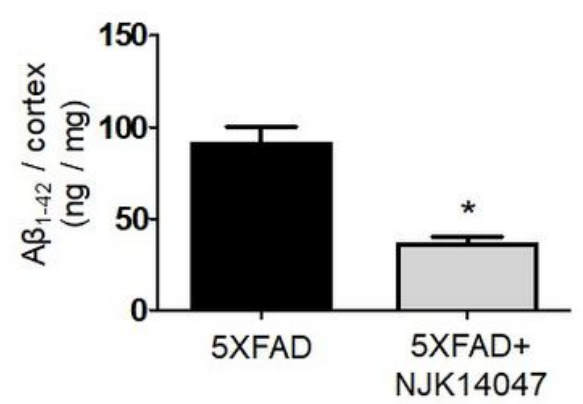

Hippocampus

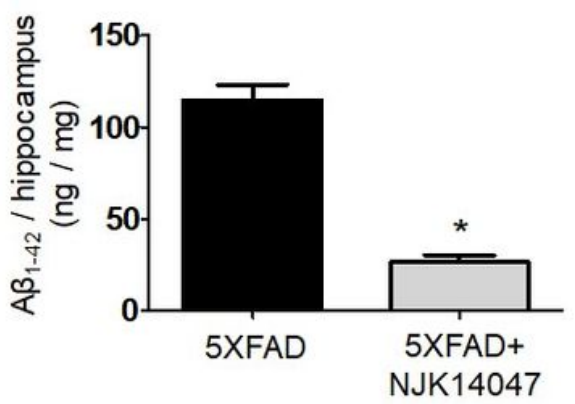

Figure 2 
NJK14047 treatment from 6-month-old to 9-month-old reduces $A \beta$ plaques in cortex and hippocampus. (A and $B)$ Representative confocal microscope images of mice brain slices stained with (A) thioflavin $S$ dye and (B) $6 \mathrm{E} 10$ antibody. Thioflavin S positive area per square millimeter and $6 \mathrm{E} 10$ positive area per square millimeter was quantified. For quantification, 4 areas in each section and 4 sections in each mouse were used. Data are shown as mean \pm SEM (scale bar $=100 \mu \mathrm{m}, \mathrm{n}=5$ per group, One-way ANOVA analysis). (C) $A \beta$ protein level per brain cortex and hippocampus weight $(\mathrm{mg})$ was measured using A $31-42$ ELISA. Data are shown as mean \pm SEM $(n=6$ per group, Student's $t$-test). (\#P $<0.05$ vs. wild type; $* P<0.05$ vs. 5XFAD) 


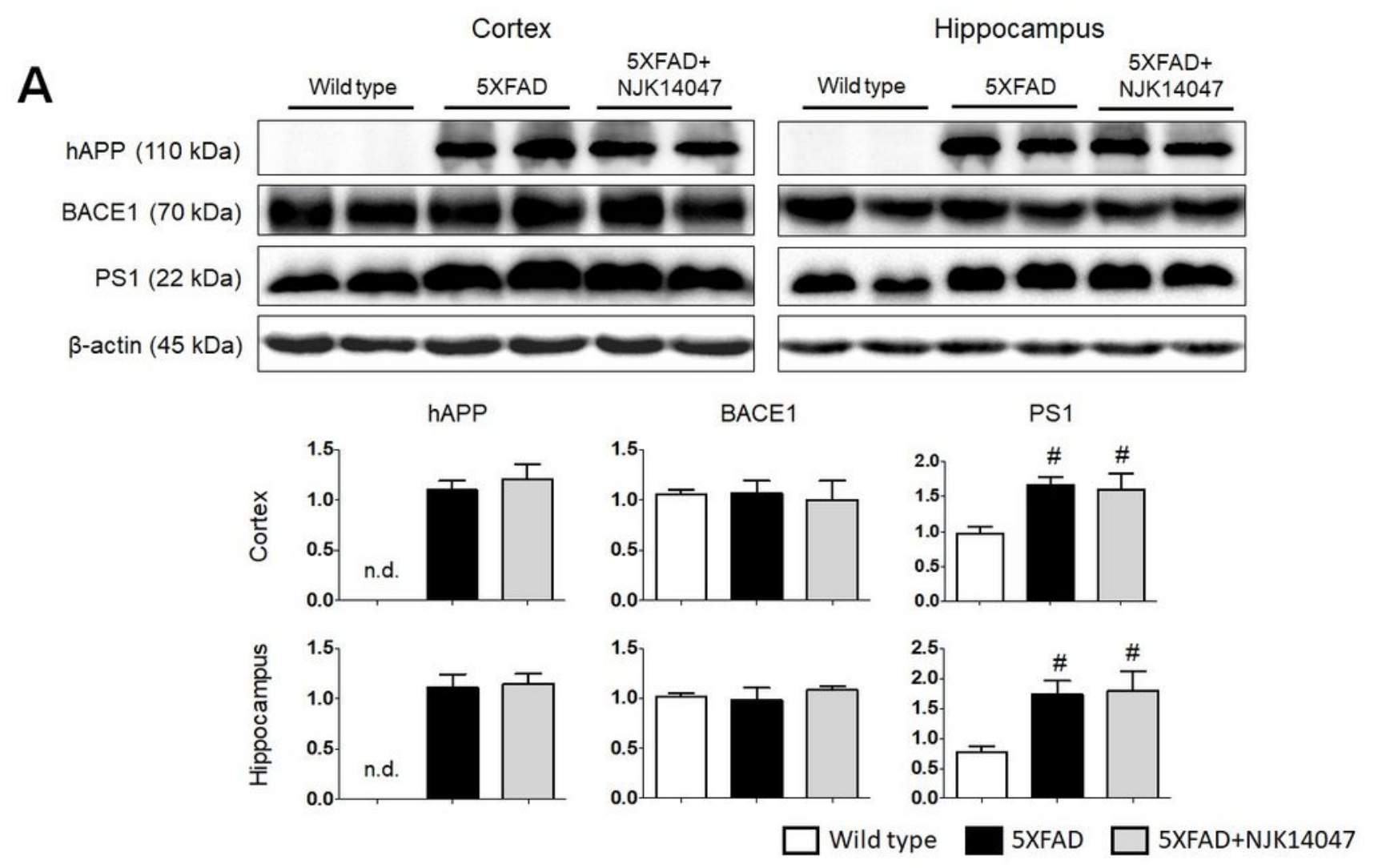

B

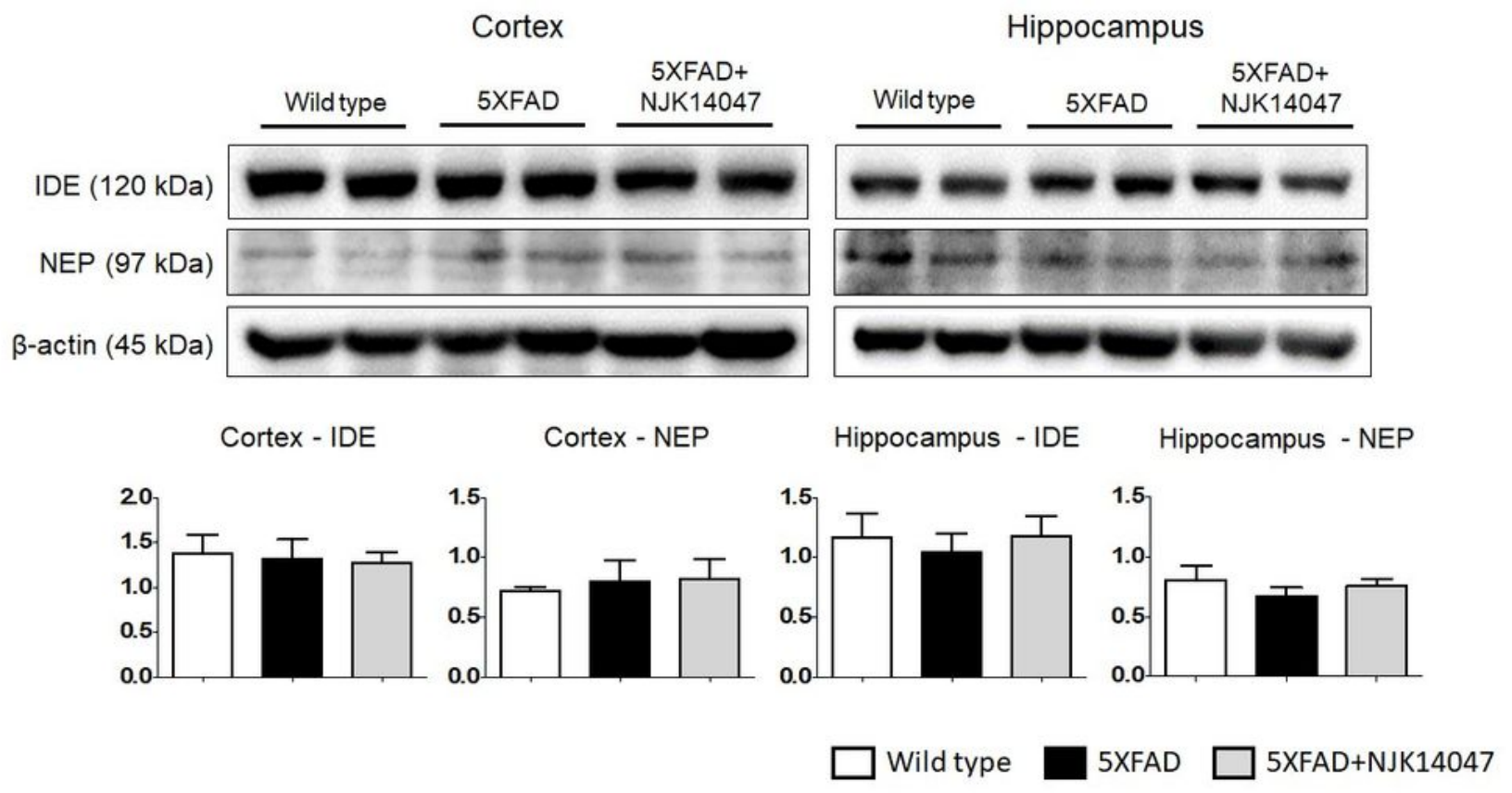

\section{Figure 3}

NJK14047 treatment from 6-month-old to 9-month-old does not effect on amyloid beta precursor protein processing and degrading molecules. (A) Representative immunoblotting images and quantifications for proteins involved in the production of $A \beta$. Total protein lysates of cortex and hippocampus were used and immunoblotted with 6E10, BACE1 and PS1 antibodies. Protein levels were quantified using image $\mathrm{J}$ and normalized with its $\beta$-actin level. Data were shown as mean \pm SEM ( $n=5$ per group, One-way ANOVA 
analysis). (B) Representative immunoblotting images and quantifications for proteins involved in the elimination of $A \beta$. Immunoblot was carried out with IDE and NEP antibodies using total protein lysates of cortex and hippocampus. Quantitative analysis was performed using image J. Protein levels were normalized to $\beta$-actin and expressed as mean \pm SEM ( $n=5$ per group, One-way ANOVA analysis).

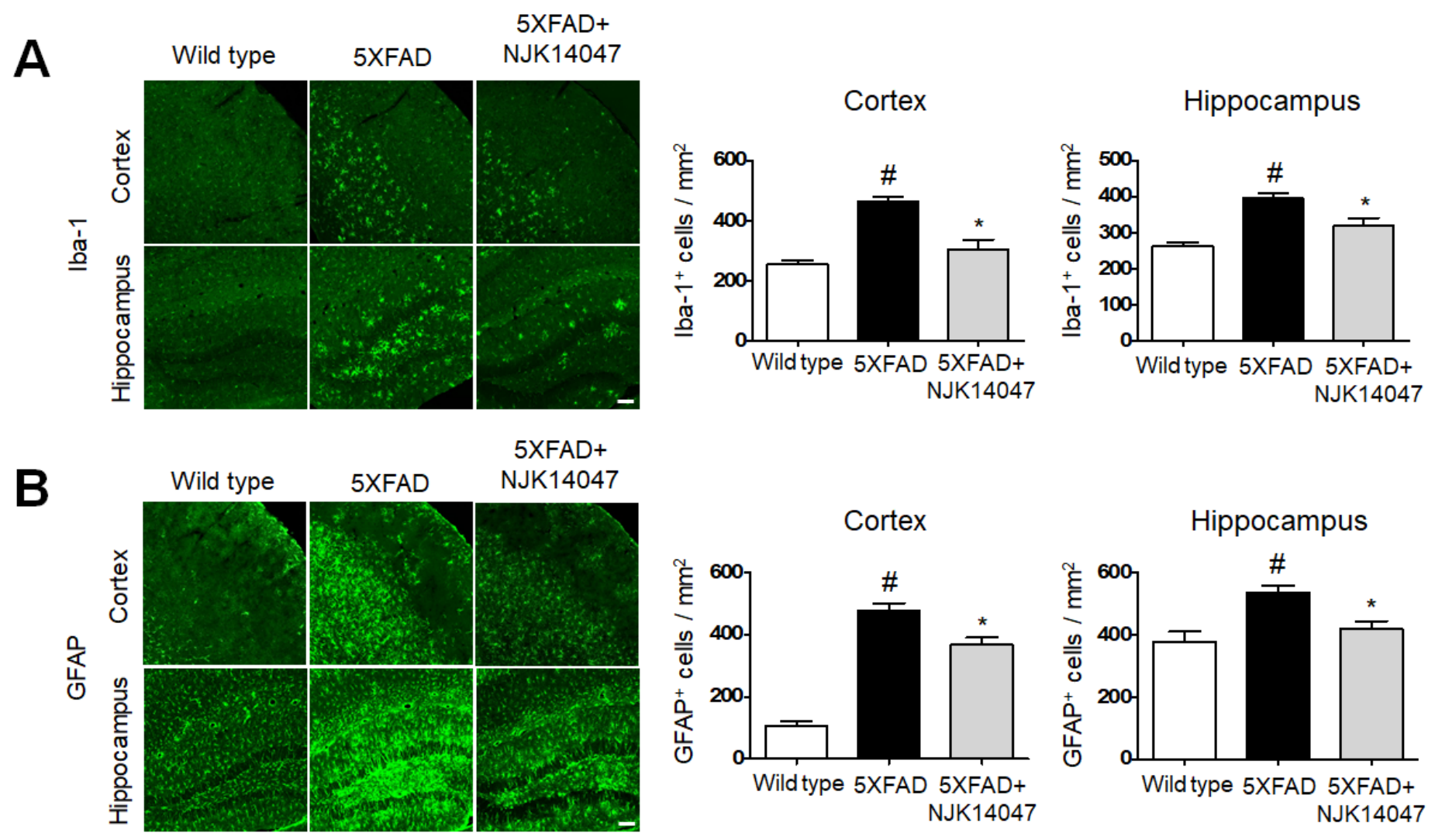

C

Cortex

Hippocampus
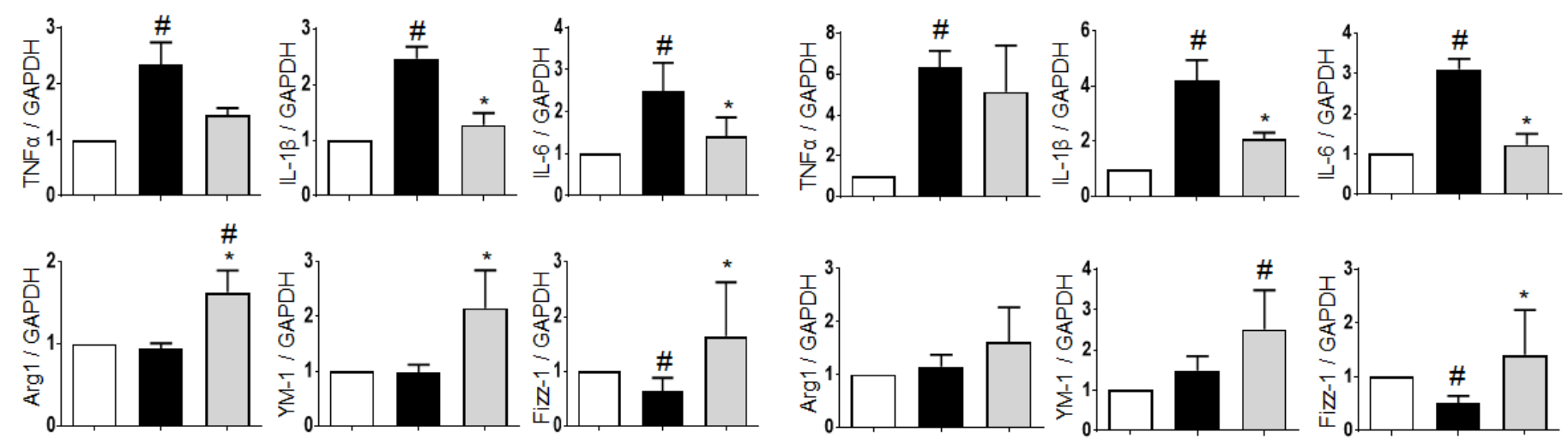

Figure 4 
NJK14047 treatment from 6-month-old to 9-month-old down-regulates neuroinflammation in cortex and hippocampus. (A and B) Representative confocal microscope images of mice brain sections stained with (A) Iba-1 antibody for labeling microglia and (B) GFAP antibody for labeling astrocytes. Iba-1 and GFAP positive cell numbers per square millimeter were measured. Data are shown as mean \pm SEM (scale bar $=$ $100 \mu \mathrm{m}, \mathrm{n}=5$ per group, One-way ANOVA analysis). (C) Relative mRNA expression levels of M1 and M2 microglial markers were measured using qRT-PCR. Total mRNA was extracted in the mice cortex and hippocampus. The mRNA expression levels were normalized with GAPDH and represented as fold of control mean $\pm S D\left(n=5-6\right.$ per group, Kruskal-Wallis test). (\#P<0.05 vs. wild type; ${ }^{*}<0.05$ vs. 5XFAD) 
A
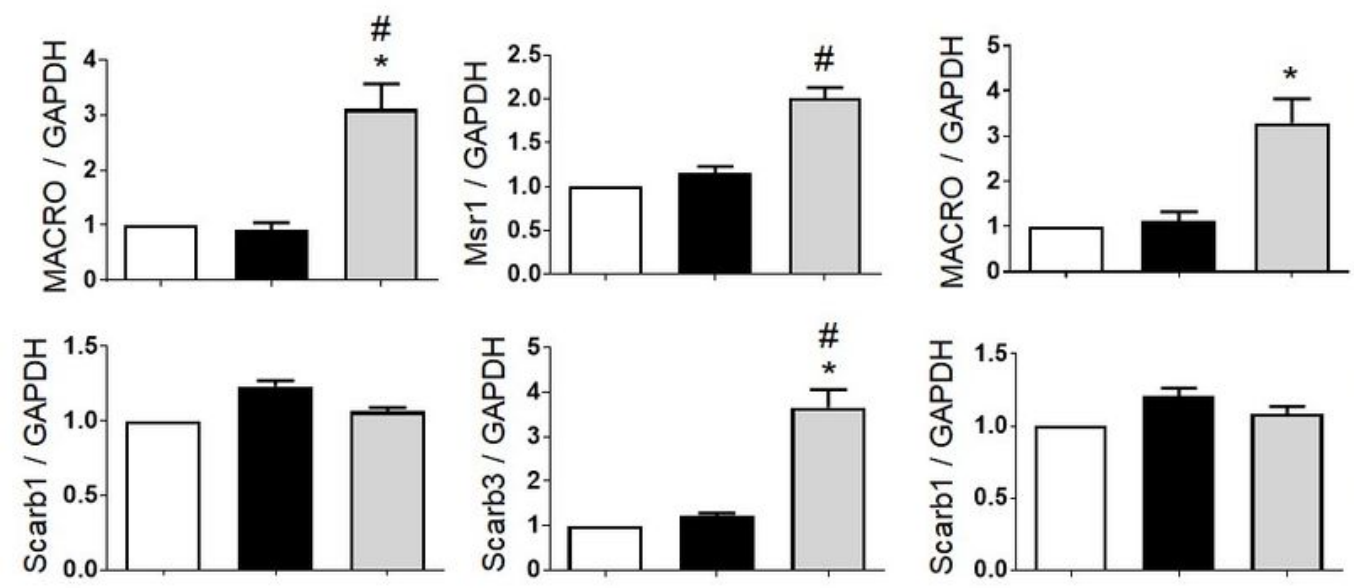

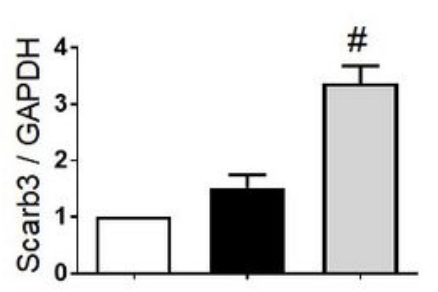

Wild type

5XFAD $\square$ 5XFAD+NJK14047

Hippocampus
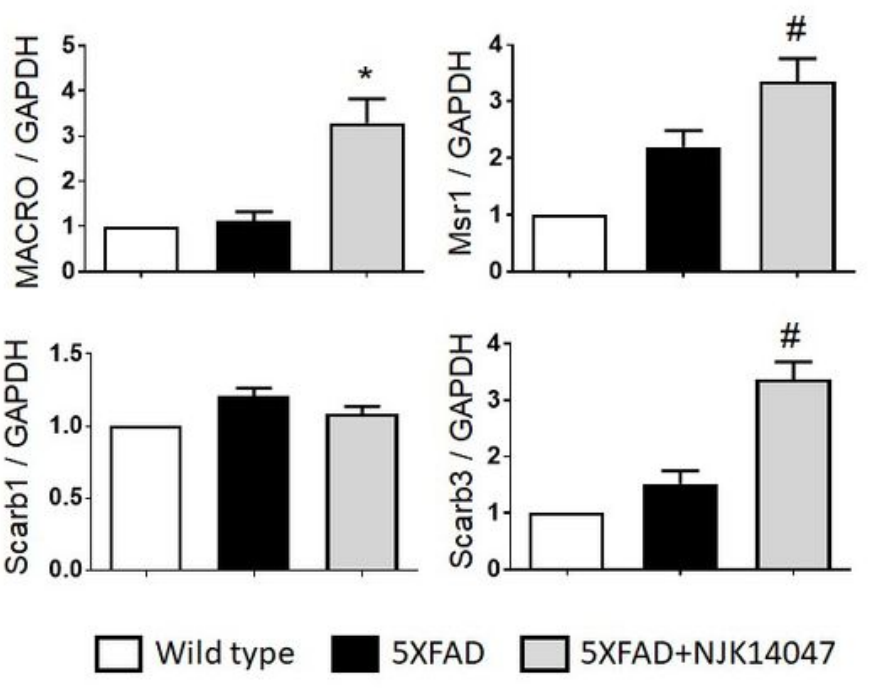

Thioflavin S
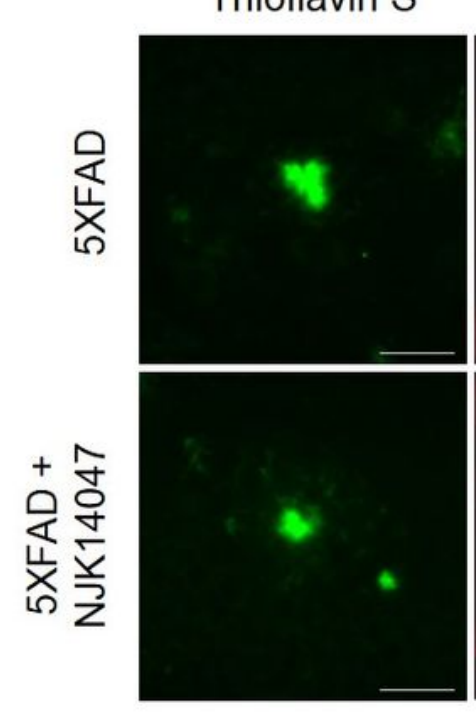

Iba-1
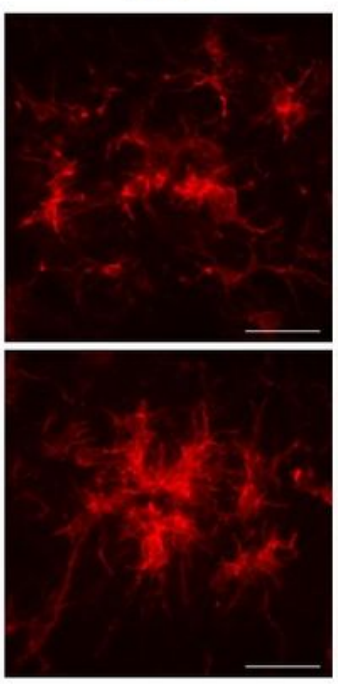
stained with thioflavin $S$ and Iba-1 antibody (Scale bar $=30 \mu \mathrm{m}$ ). The number of plaque associated microglial cells within $20 \mu \mathrm{m}$ range of $A \beta$ plaque were quantified. Data were shown as mean $\pm S E M(n=5$ per group, Student's t-test). (\#P $<0.05$ vs. wild type; *P $<0.05$ vs. 5XFAD)

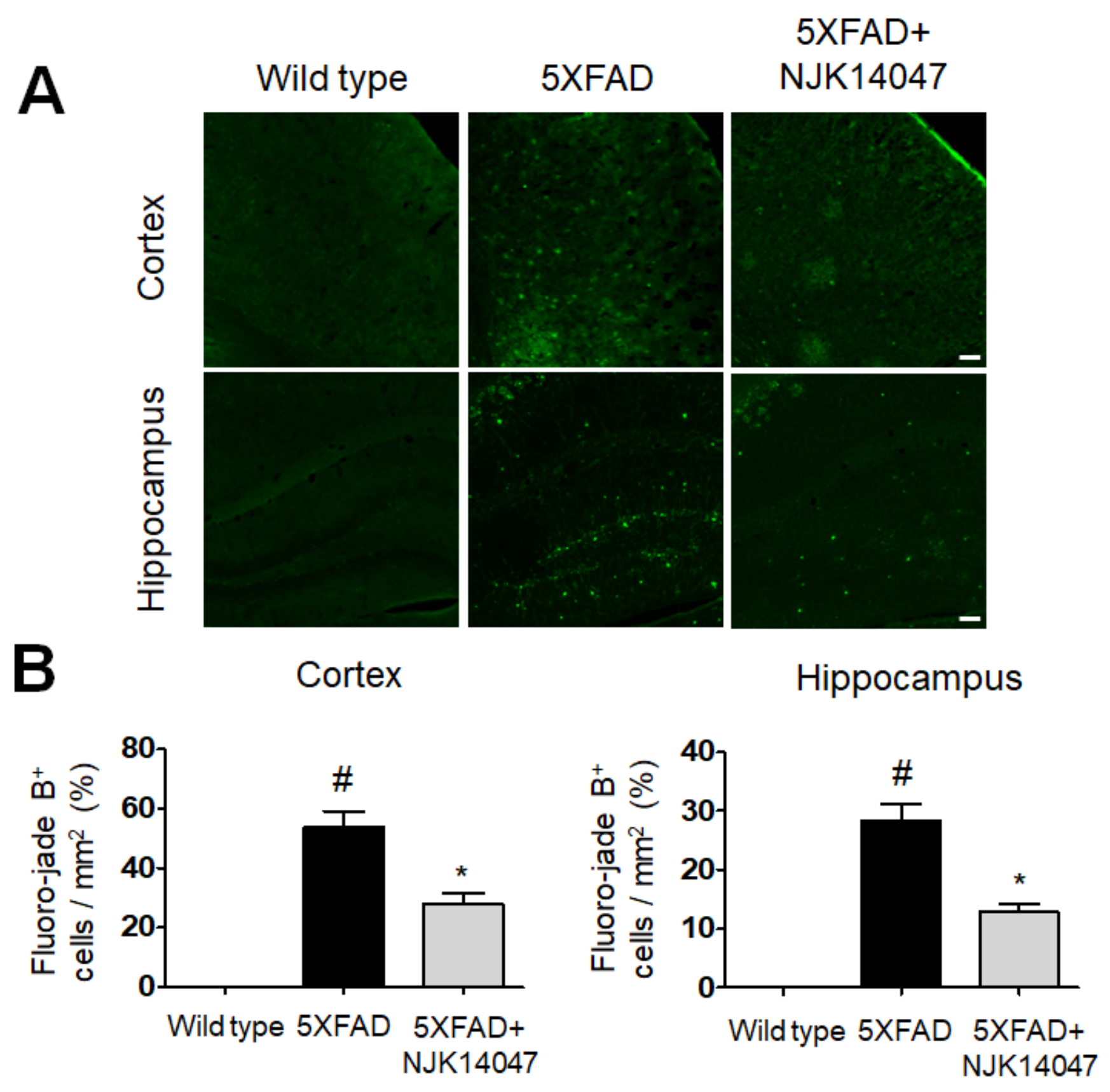

Figure 6

NJK14047 treatment from 6-month-old to 9-month-old inhibits neuronal deaths in cortex and hippocampus. (A) Representative confocal microscope images of mice brain sections stained with Fluoro-jade B dye for labeling degenerating neurons in cortex and hippocampus. (B) Quantitative analysis 
was performed with 4 sections in each mouse and 4 areas in each section. Fluoro-jade B positive cells per square millimeter were measured. Data are shown as mean \pm SEM (scale bar $=100 \mu \mathrm{m}, \mathrm{n}=5$ per group, One-way ANOVA analysis). (\#P $<0.05$ vs. wild type; *P $<0.05$ vs. 5XFAD)

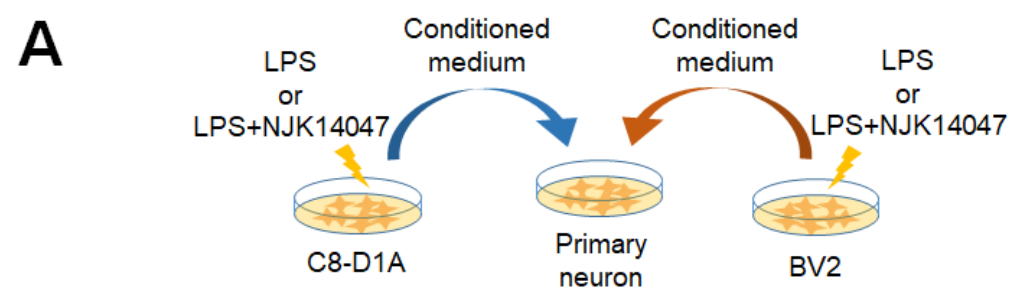

B
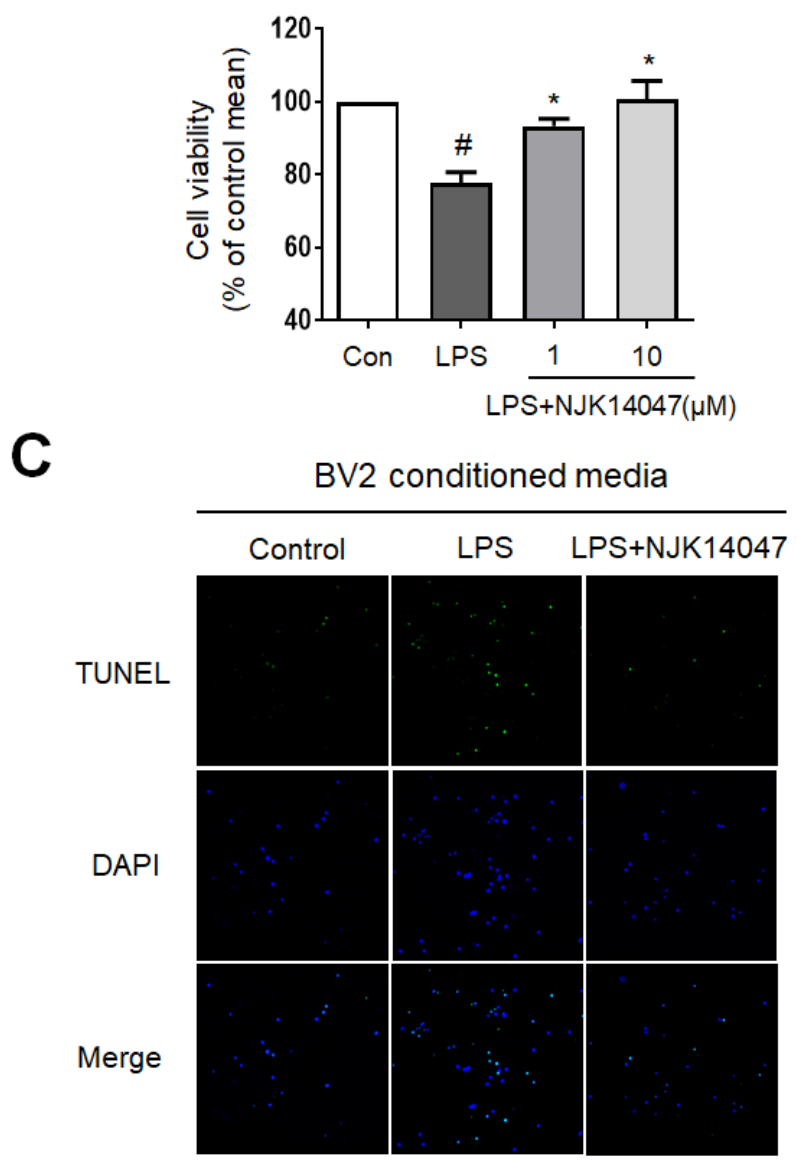

BV2 conditioned media

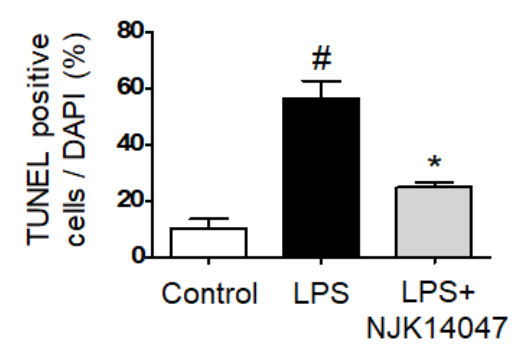

C8-D1A CM to neuron

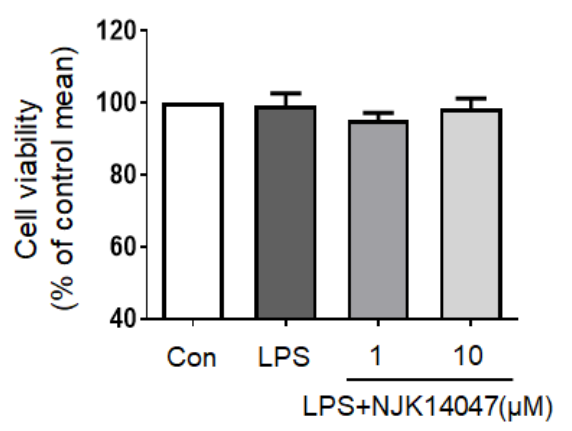

C8-D1A conditioned media

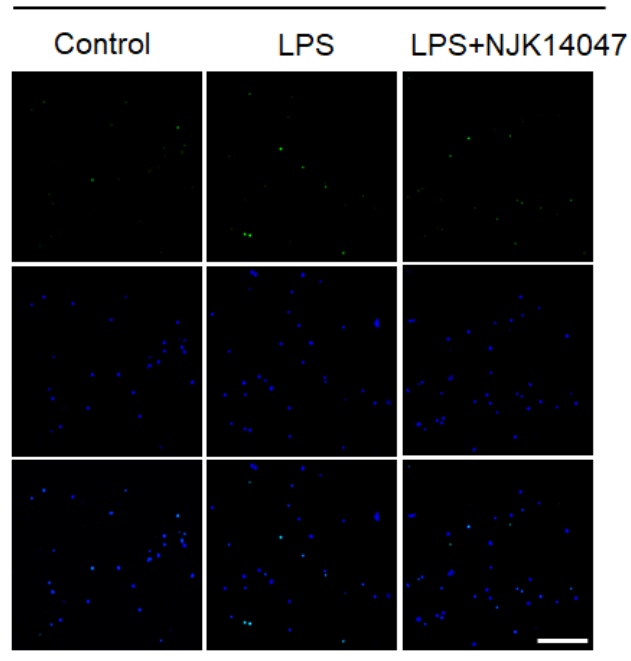

C8-D1A conditioned media

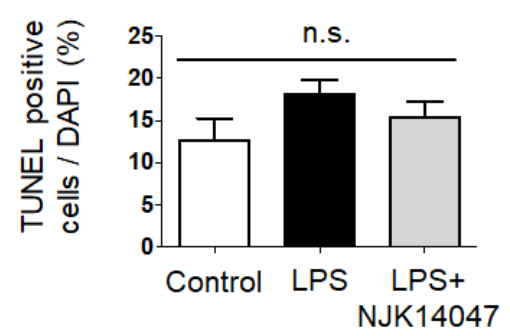

Figure 7

NJK14047 attenuates neurotoxicity of LPS-stimulated microglial cell line rather than that of LPSstimulated astrocytes cell line. (A) Scheme of the experiment. Microglial cell line BV2 and astrocyte cell 
line C8-D1A were seeded on 6-well plate at 5×105 cells/well. BV2 and C8-D1A were stimulated with 500 $\mathrm{ng} / \mathrm{ml}$ LPS after $2 \mathrm{~h}$ of pre-treatment of 1 or $10 \mu \mathrm{M}$ NJK14047. After $22 \mathrm{~h}$ of LPS stimulation, all medium was changed to fresh Neurobasal medium in which cells were incubated for $24 \mathrm{~h}$. The conditioned medium was collected and treated to primary neurons seeded on 48-well plate for cytotoxicity assay (B) or 24-well cover glass for TUNEL assay (D). (B) Viability of mice primary neurons stimulated with BV2 and C8-D1A conditioned media. O.D. at $570 \mathrm{~nm}$ were normalized to control group and shown as $\%$ of control mean $\pm S D$ ( $n=3$, Kruskal-Wallis test) (C) TUNEL assay in mice primary neurons stimulated with BV2 and C8-D1A conditioned media. Apoptotic cells were quantified using 4 images in each cover glass. Data are shown as mean \pm SEM (scale bar $=100 \mu \mathrm{m}, \mathrm{n}=5$, One-way ANOVA analysis). (\#P $<0.05$ vs. control group; ${ }^{P}<0.05$ vs. LPS group) 


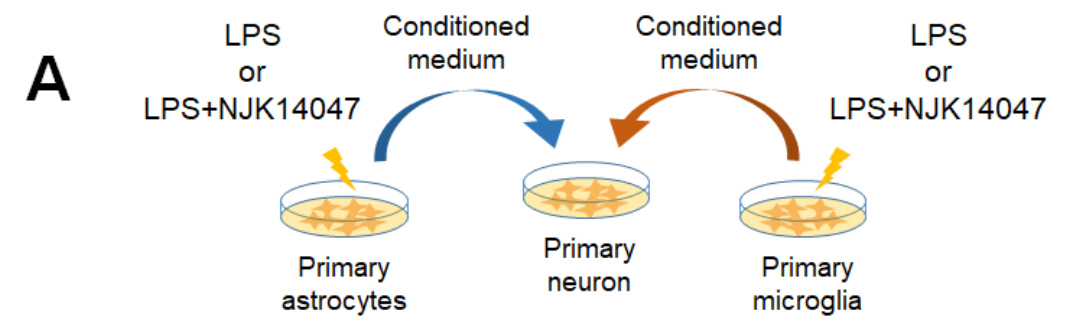

B

MCM to neuron

ACM to neuron
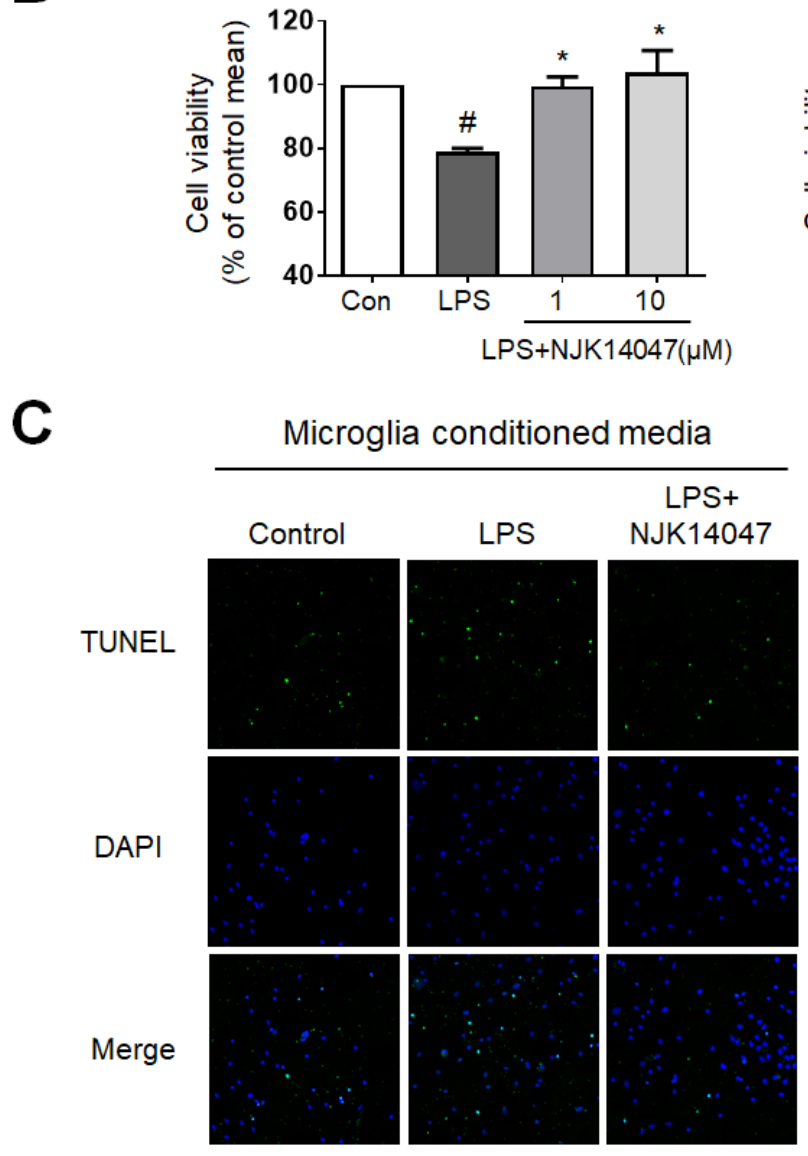

Microglia conditioned media
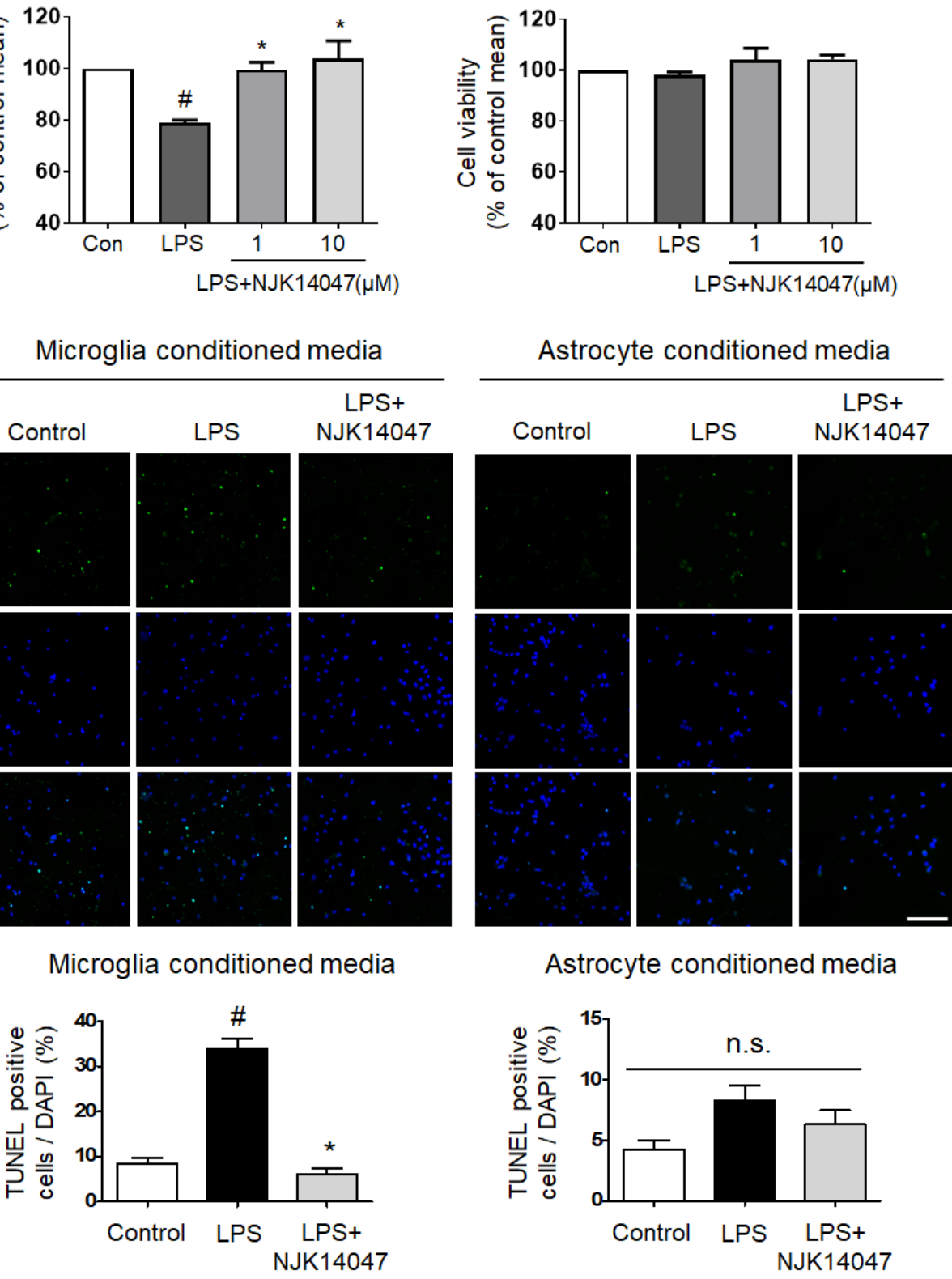

Astrocyte conditioned media

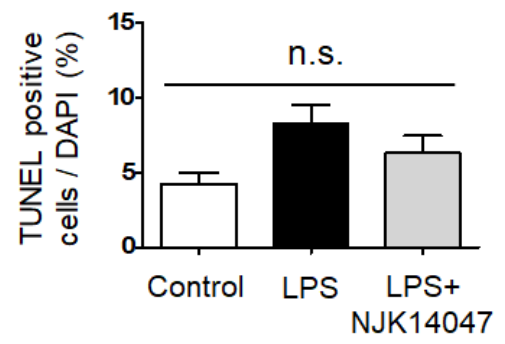

\section{Figure 8}

NJK14047 reduces LPS-stimulated microglial neurotoxicity rather than LPS-stimulated astrocytes neurotoxicity. (A) Scheme of the experiment. Mice primary microglia and astrocytes were seeded on 6well plate at $5 \times 105$ cells/well and stimulated with $50 \mathrm{ng} / \mathrm{ml}$ LPS after $2 \mathrm{~h}$ of pre-treatment of $10 \mu \mathrm{M}$ NJK14047. After $22 \mathrm{~h}$ of LPS stimulation, all medium was changed to fresh Neurobasal medium in which cells were incubated for $24 \mathrm{~h}$. The conditioned medium was collected and treated to primary neurons 
seeded on 24-well cover glass for TUNEL assay. (B) Viability of mice primary neurons incubated with MCM and ACM for 24 h. O.D. at $570 \mathrm{~nm}$ were normalized to control group and shown as $\%$ of control mean $\pm S D$ ( $n=3$, Kruskal-Wallis test) (C) TUNEL assay in mice primary neurons stimulated with mice primary microglia and astrocytes conditioned media. Apoptotic cells were quantified using 4 images in each cover glass. Data are shown as mean \pm SEM (scale bar $=100 \mu \mathrm{m}, \mathrm{n}=5$, One-way ANOVA analysis). (\#P $<0.05$ vs. control group; $* \mathrm{P}<0.05$ vs. LPS group) 